Dante Vailati*

\title{
Revisione tassonomica delle «serie filetiche di Dellabeffaella e di Boldoria» con descrizione di quattro nuovi generi (Coleoptera Cholevidae Leptodirinae)
}

\begin{abstract}
Riassunto: Nel presente lavoro l'autore affronta la revisione critica dell'assetto sistematico e tassonomico dei generi Boldoria Jeannel, $1924 \mathrm{e}$ Pseudoboldoria Ghidini, 1937, alla luce di una nuova interpretazione del valore informativo di alcuni caratteri discriminanti, usati in passato, in modo prudenziale, semplicemente per distinguere al loro interno diversi "gruppi di specie". Alcuni di tali gruppi vengono ora proposti, riconoscendone le profonde differenze, al rango di generi distinti.

Al genere Boldoria Jeannel, 1924 viene mantenuta la sola specie aculeata Jeannel, 1924. Viene riabilitato il genere Ghidinia Pavan, 1939 per le specie aguinensis Vailati, 1974, morettii Pavan, 1939 e vailatii Cavadini, 1988. Viene riabilitato, e considerato genere distinto, Hartigiella Müller, 1935 per la specie baldensis Müller, 1928. Vengono inoltre descritti i seguenti nuovi generi: Pavaniola n. gen. per le specie regalini Vailati, 1988, comottii Vailati, 1988, longitarsis Pavan, 1941 e ghidinii Lona, 1937 con relative subspecie; Cacciamalia $\mathrm{n}$. gen. per le specie allegrettii Jeannel, 1930, antonellae Vailati, 1988, viallii Pavan, 1938 e glacialis Vailati, 1975 con relative subspecie; Ragazzonia n. gen. per le specie breviclavata Müller, 1931, trumplina Vailati, 1988 e vestae Ghidini, 1936 con relative subspecie.

Al genere Pseudoboldoria Ghidini, 1937 sono assegnate le specie barii Focarile, 1950, bergamasca (Jeannel, 1914), kruegeri (Müller, 1914) con relative subspecie, intermedia Vailati, 1988, longobarda Vailati, 1988, gratiae Monguzzii, 1984 e belluccii Regalin, 1985. Viene inoltre descritto il genere Stoppaniola n. gen. per le specie robiati (Reitter, 1889), personata Vailati, 1988, focarilei Vailati, 1988, malanchinii Pavan \& Ronchetti, 1949, bucciarellii Rossi, 1963, schatzmayri Focarile, 1952 e comottiana Vailati, 1988.

Viene quindi analizzata la distribuzione dei generi qui trattati e sono discusse le reciproche affinità. Per i generi Boldoria Jeannel, 1924, Pavaniola n. gen., Cacciamalia n. gen., Hartigiella Müller, 1935, Ghidinia Pavan, 1939, Ragazzonia n. gen., Viallia Pavan, 1950, Insubriella Vailati, 1990, Monguzziella Vailati, 1993 cui si aggiunge Miettiella Piva, 2016 di recente descrizione viene confermata la loro appartenenza alla «serie filetica di Boldoria», mentre ne viene considerato estraneo il genere Cryptobathyscia Vailati, 1980, cui in via provvisoria vengono riconosciute affinità che saranno da ricercare all'interno dell'eterogeneo "complesso Bathysciola", come fino ad oggi inteso, quando finalmente venisse sottoposto ad una necessaria revisione. Per i generi Canavesiella Giachino, 1993, Dellabeffaella Capra, 1924, Archeoboldoria Ghidini, 1937, Pseudoboldoria Ghidini, 1937 e Stoppaniola n. gen. viene confermata l'appartenenza alla «serie filetica di Dellabeffaella».

In conclusione vengono discusse le evidenze biogeografiche del complesso trattato sulla base delle attuali conoscenze. Diversi modelli di distribuzione e diffusione di generi e specie nell'area prealpina sarebbero connessi a vicende paleogeografiche e paleoclimatiche di periodi di diversa durata.
\end{abstract}

\footnotetext{
Abstract: Taxonomic revision of the «phyletic lineages of Boldoria and Dellabeffaella» with description of four new genera (Coleoptera Cholevidae Leptodirinae).

This contribution deals with the critical revision of taxonomy and systematics of the genera Boldoria Jeannel, 1924 and Pseudoboldoria Ghidini, 1937. It includes a new interpretation of some diacritic characters, used in the past, to differentiate several "species groups". Some of these groups are now upgraded to the rank of genera.

The genus Boldoria Jeannel, 1924 is maintained for B. aculeata Jeannel, 1924 only. The genus Ghidinia Pavan, 1939 is restored for the species G. aguinensis Vailati, 1974, G. morettii Pavan, 1939 and G. vailatii Cavadini, 1988. Hartigiella Müller, 1935 is restored, and considered a distinct genus, for $H$. baldensis Müller, 1928. The following new genera are described: Pavaniola n. gen. including P. regalini Vailati, $1988, P$. comottii Vailati, 1988, P. longitarsis Pavan, 1941, and P. ghidinii Lona, 1937 with related subspecies; Cacciamalia n. gen. including C. allegrettii Jeannel, 1930, C. antonellae Vailati, 1988, C. viallii Pavan, 1938 and C. glacialis Vailati, 1975 with related subspecies; Ragazzonia n. gen. including $R$. breviclavata Müller, 1931, $R$. trumplina Vailati, 1988 and $R$. vestae Ghidini, 1936 with related subspecies.

The genus Pseudoboldoria Ghidini, 1937 is maintained with the species P. barii Focarile, 1950, P. bergamasca (Jeannel, 1914), P. kruegeri (Müller, 1914) with its subspecies, P. intermedia Vailati, 1988, P. longobarda Vailati, 1988, P. gratiae Monguzzi, 1984 and P. belluccii Regalin, 1985. The genus Stoppaniola n. gen. is also described including S. robiati (Reitter, 1889), S. personata Vailati, 1988, S. focarilei Vailati, 1988, S. malanchinii Pavan \& Ronchetti, 1949, S. bucciarellii Rossi, 1963, S. schatzmayri Focarile, 1952 and S. comottiana Vailati, 1988.

The distribution of the genera is analyzed and their reciprocal affinities are proposed and discussed. The genera Boldoria Jeannel, 1924, Pavaniola n. gen., Cacciamalia n. gen., Hartigiella Müller, 1935, Ghidinia Pavan, 1939, Ragazzonia n. gen., Viallia Pavan, 1950, Insubriella Vailati, 1990, Monguzziella Vailati, 1993 and Miettiella Piva, 2016 are confirmed in the "phyletic lineage of Boldoria», while Cryptobathyscia Vailati, 1980, is provisionally placed into the heterogeneous "Bathysciola complex", waiting for a complete revision. The genera Canavesiella Giachino, 1993, Dellabeffaella Capra, 1924, Archeoboldoria Ghidini, 1937, Pseudoboldoria Ghidini, 1937 and Stoppaniola n. gen. are confirmed in the «phyletic lineage of Dellabeffaella».
}

*Dante Vailati, Via Interna 8, 25127 Brescia, Italy. E-mail: dante.vailati@libero.it 
The last part deals with the biogeographical aspects of the taxonomic complex on the basis of current knowledge. Some patterns about the actual distribution and spreading of genera and species in the Prealpine area would be connected with paleogeographic and paleoclimatic events of different duration periods.

Key words: Cholevidae; Leptodirinae; New genera; taxonomy; biogeography; Central Pre-Alps.

\section{INTRODUZIONE}

L'ultimo lavoro d'insieme che tratta in modo organico della sistematica del genere Boldoria Jeannel, dovuto allo scrivente, risale ormai a quasi tre decenni addietro (Vailati, 1988). In tale sede non solo erano rivedute la sistematica e la tassonomia dei taxa fino ad allora noti, elevando i sottogeneri Boldoria, Pseudoboldoria e Archeoboldoria, come intesi da Ghidini (1937) al rango di generi distinti, ma venivano descritte numerose specie e sottospecie nuove per la Scienza, che accrescevano in modo considerevole, rispetto a quanto noto dai lavori precedenti, le conoscenze non solo tassonomiche, ma anche sulla distribuzione di questi Leptodirinae diffusi nella fascia comprendente le Alpi Biellesi e le intere Prealpi Lombarde (nel senso della SOIUSA, Marazzi, 2005), aspetto quest'ultimo sostenuto dalle molte nuove stazioni, nel frattempo censite da vari ricercatori in molti anni di ricerche sul campo.

All'epoca del lavoro citato (Vailati, 1988), in particolare per quanto riguarda il genere Boldoria, era già stata evidenziata al suo interno una certa disomogeneità, sottolineata dall'aver individuato fra le specie note, già allora, differenze e affinità tali da suggerire l'esistenza di complessi naturali che l'autore preferiva "prudenzialmente" definire come "gruppi di specie", spiegando le ragioni per le quali non riteneva fosse il momento di formalizzare dei raggruppamenti di categoria sottogenerica, ritenendoli prematuramente inopportuni, nella convinzione che vi fossero «... ancora diverse lacune nel nostro quadro conoscitivo speciografico» (Vailati, 1988, p. 26).

In una premessa allo stesso lavoro citato - dopo aver ricordato come Boldoria fosse stato in origine istituito da Jeannel (1924) come sottogenere di Bathysciola, quindi elevato a genere da Ghidini (1937) e suddiviso nei tre sottogeneri Archeoboldoria, Pseudoboldoria e Boldoria, sottogeneri che infine, in quella sede (Vailati, 1988), si apprestava a elevare al rango di generi distinti - l'autore scriveva: «... Una continua, inevitabile elevazione di rango che trova giusti- ficazione in un sempre maggior approfondimento delle conoscenze, in un sempre maggior numero di specie note $e$, in definitiva, in una più chiara visione della distanza tra $i$ vari taxa e quindi dell'eterogeneità di ciò che un tempo poteva sembrare un raggruppamento omogeneo», parole che oggi ci sembrano superflue poiché scontato è il loro significato, ma sentite allora doverose per giustificare in qualche modo quella che in realtà altro non era che la naturale evoluzione delle conoscenze. Non possiamo nascondere che trent'anni fa poteva sembrare quasi un azzardo frammentare un genere Boldoria che era stato trattato come tale per decenni, ma vale la pena ricordare che tale lettura dei fatti era anche l'esito di un retaggio culturale che imponeva molta cautela nel "toccare" impostazioni consolidate nel tempo. Non solo la pretesa omogeneità di un genere che "doveva" essere distribuito su una certa area delle Prealpi Lombarde, ma soprattutto la valutazione dei caratteri che lo definiva, considerati nel loro insieme per accomunarvi tutte le specie allora note in un unico taxon, fu la principale ragione che rese inevitabili certe scelte, non ultima quella di includervi pure il genere Ghidinia Pavan e, prima ancora, Bathysciola (Hartigiella) baldensis Müller (Vailati, 1976), non ravvisandovi, nell'ottica del momento, caratteri sufficienti per discriminarli in generi distinti.

Ciò nonostante, le differenze che caratterizzavano i "gruppi di specie" allora istituiti, apparivano già all'epoca abbastanza profonde, anche alla luce di alcuni caratteri che venivano presi in considerazione come discriminanti per la prima volta, come la morfologia degli speroni tibiali dentati, il metendosternite (organo di Crowson) e il processo metanotale.

Sono di anni successivi le descrizioni di nuovi taxa generici e specifici, quali Insubriella paradoxa delle Prealpi Bresciane (Vailati, 1990) e Monguzziella grottoloi delle Prealpi Venete (Vailati, 1993a), che l'autore assegna alla «serie filetica di Boldoria», oltre a due specie del nuovo genere Canavesiella delle Alpi Graie nord-orientali (Giachino, 1993a) e tre nuove specie di Archeoboldoria (Giachino \& Vailati, 1997; Giachino, Lana \& Vailati, 2001; Casale \& Giachino, 2010) delle 
Alpi Graie e Pennine, ascrivibili queste alla «serie filetica di Dellabeffaella».

Se si escludono questi recenti e pur importanti apporti, va rilevato che in questi ultimi decenni non vi è stata la scoperta nelle Prealpi Lombarde di alcun nuovo taxon riferibile ai generi Boldoria e Pseudoboldoria. L'auspicato incremento del «quadro conoscitivo speciografico» dunque è stato in linea generale modesto e, per quanto riguarda questi due generi, non vi è stato per nulla, se si esclude il fatto che, grazie alle ricerche che nell'area interessata sono proseguite senza sosta e con una certa intensità, non solo da parte dello scrivente, si è raccolta una notevole massa di nuovi dati geonemici per una sempre migliore definizione degli areali e dell'ecologia delle specie note (Giachino \& Vailati, 1997, 2005, 2008; Vailati, 1991, 1993b); inoltre, l'aver potuto esaminare abbondanti materiali provenienti da molte stazioni, ci permette anche di individuare oggi nuovi taxa specifici che in passato non erano stati riconosciuti come tali e che formeranno oggetto di un ulteriore contributo. Ciò contrariamente a quanto è avvenuto per diversi generi di Leptodirinae delle Prealpi Venete e Carniche che, proprio negli anni a cavallo tra la fine dello scorso secolo e l'inizio dell' attuale, hanno visto un notevole incremento delle conoscenze, fra le quali sono da annoverare la descrizione di poco meno di una ventina di nuovi taxa (Piva, 1993, 2000, 2005; Giachino \& Vailati, 2005), cui si aggiunge la recentissima scoperta del nuovo genere e nuova specie Miettiella vespertilio nei Lessini veronesi (Piva, 2016). Tuttavia, l'opportunità di studiare abbondanti materiali della fauna a Cholevidae, in particolare per quanto concerne i Leptodirinae, in un più ampio quadro coinvolgente l'intero arco alpino, fra i quali si possono mettere a confronto le analogie e le differenze di svariate situazioni, ha prodotto in tutti questi anni maggiore consapevolezza della complessità riguardante la sistematica dei vari raggruppamenti, le affinità tra loro intercorrenti, il valore e il peso degli stessi caratteri morfologici utilizzati per accomunarli o per separarli, la loro stessa distribuzione, anche in termini di ampiezza degli areali e il valore informativo dovuto all'insieme di tutti questi aspetti, in altre parole una maggiore chiarezza sullo stesso significato di tutto ciò quale chiave per una nuova lettura dei livelli tassonomici di volta in volta individuati.

L'originaria scelta di considerare i raggruppamenti trattati come informali "gruppi di specie" è sempre stata accompagnata nello stesso scrivente, come già evidenziato, dalla constatazione dell'esistenza di profonde differenze fra i "gruppi" stessi, tali da richiedere, oggi, di essere contestualizzate attraverso una revisione più ponderata, basata sull'analisi critica dei caratteri fin qui utilizzati, ancorché di altri in passato trascurati. Non solo, ma l'analisi effettuata in questo nuovo quadro porta inevitabilmente a riconsiderare le affinità intrageneriche che, viste sotto una nuova luce, rimettono in discussione anche l'appartenenza o meno dei generi trattati alle «serie filetiche» come in precedenza definite e alla loro stessa validità.

Il risultato di questa analisi è la proposta di istituire alcuni nuovi generi per assegnare ad alcuni "gruppi di specie", allora designati come tali, e come tali attribuiti ai generi Boldoria e Pseudoboldoria (Vailati, 1988), il rango di generi distinti. Al contempo vengono riabilitati, nella loro validità di generi distinti, Ghidinia Pavan, 1939 e Hartigiella Müller, 1935, che erano stati attribuiti "forzatamente" al genere Boldoria per le ragioni sopra esposte. Altrettanto inopportuno, e non privo di dubbi fin dall'inizio, era stato l'inserimento nella «serie filetica di Boldoria» del genere Cryptobathyscia Vailati, 1980 (Sbordoni et al., 1982), taxon che appare oggi, se non geograficamente, morfologicamente e tassonomicamente alquanto isolato, che viene quindi escluso dalla presente trattazione e per la cui corretta valutazione bisognerà attendere l'auspicata revisione delle specie italiane di quella galassia che costituisce l'eterogeneo genere Bathysciola come ancora oggi inteso dagli Autori.

Scopo principale di questo contributo, dopo la chiave dicotomica per la determinazione dei generi, è di fornire la descrizione o ridescrizione di tutti i generi - qui considerati sensu novo e assegnati alle "serie filetiche di Dellabeffaella e di Boldoria" - integrata ove necessario con nuovi elementi utili ad una corretta discriminazione. All'interno di ogni genere sono semplicemente elencate le specie note ad esso assegnate (raggruppate ancora, ove necessario, in "gruppi di specie"), per le quali si è giudicato superfluo fornire sia le singole descrizioni sia la relativa iconografia, in quanto si ritengono sufficientemente accurate quelle già contenute nei lavori originali, o comunque precedenti, ai quali si rimanda.

\section{Materiali e MEtodi}

I materiali utilizzati per la presente revisione, ove non diversamente indicato, sono conservati nella col- 
lezione dell'autore. Per l'analisi dei caratteri mi sono avvalso sia di nuovi preparati appositamente allestiti sia dell'abbondante materiale iconografico presente nel mio archivio. I nuovi preparati, tutti montati in Balsamo del Canada su cartellini trasparenti e disegnati alla camera lucida montata su microscopio Leitz Dialux, riguardano soprattutto caratteri morfologici che in passato erano stati trascurati.

\section{Scelta dei caratteri}

In linea generale, vengono considerati i caratteri già utilizzati in passato nella loro validità a livello di genere e di "gruppi di specie" e già ampiamente discussi in precedenza (Vailati, 1988), dando per questi ultimi semplicemente una diversa lettura nel loro valore discriminante. Le profonde differenze esistenti, soprattutto evidenziate dalle morfologie dei genitali maschili, vengono enfatizzate dall'accostamento di altri caratteri, come quelli pertinenti alle femmine, che ne perfezionano le diagnosi di livello generico.

A questo punto è necessario riassumere alcune osservazioni relative ai caratteri presi in considerazione, in quanto in alcuni casi il loro valore discriminante rimane quello di dividere i complessi nelle due «serie filetiche di Dellabeffaella e di Boldoria». Tali sono:

Carena mesosternale. Può essere (quando ben sviluppata) dentata inferiormente all'angolo anteriore e priva di apofisi posteriore libera prolungata sul metasterno («serie filetica di Dellabeffaella») o può essere priva di dente inferiormente all'angolo anteriore e avere invece un'apofisi prolungata posteriormente a coprire il metasterno fino a superarlo («serie filetica di Boldoria»).

Apparato metatergale. Può essere grande o mediopiccolo, ma ancora poco ridotto (fa eccezione in $\mathrm{Ca}$ navesiella) nella «serie filetica di Dellabeffaella», sempre molto piccolo ed estremamente semplice nella «serie filetica di Boldoria».

Metendosternite. Può essere non scavato a V, con le braccia della furca triangolari, larghe alla base («serie filetica di Dellabeffaella») o può essere conformato a $\mathrm{V}$, con le braccia della furca libere («serie filetica di Boldoria»).

Speroni apicali interni alle tibie. Possono essere pettinati («serie filetica di Dellabeffaella») o polidentati («serie filetica di Boldoria»), come intesi da Vailati (1988).

Cestelli spinosi alle meso e metatibie. Possono essere incompleti, rudimentali, con scarse e brevi spine («serie filetica di Dellabeffaella») o completi di spine numerose, lunghe e accollate («serie filetica di Boldoria»).

Altri caratteri invece, che mostrano una certa omogeneità nella morfologia generale, possono essere presi in considerazione per differenziare, all'interno delle «serie filetiche», singoli generi. In particolare si possono osservare:

Antenne. Occorre sottolineare che la struttura delle antenne, intesa come forma e rapporto della lunghezza fra i vari antennomeri, è assai differente tra i vari generi; questo a prescindere da quelli che possono essere considerati fenomeni di specializzazione. Mentre l'allungamento generale delle antenne, infatti, può essere osservato in alcune specie più specializzate, accompagnato generalmente da un maggior allungamento del corpo (come avviene ad esempio all'interno dei generi Ghidinia, Cacciamalia n. gen., o Viallia), si può notare che la loro morfologia generale rimane omogenea all'interno dei singoli generi a prescindere dal grado di sviluppo in lunghezza (Figg. 1-29). Ad esempio, si può notare nel genere Viallia che l'antenna maschile di $V$. alfanoi (la più allungata) ha la stessa morfologia di quella di V. mismae (la più breve), con l'ultimo articolo, vagamente romboidale, sempre di pochissimo più lungo del precedente (Figg. 16-18). Anche nel genere Cacciamalia $\mathrm{n}$. gen. la morfologia antennale si presenta omogenea, a prescindere dal diverso allungamento nelle varie specie, dove si ha sempre l'ultimo antennomero allungato in modo abnorme (Figg. 13-15), ben più lungo della somma dei due precedenti, e con i lati sinuati al terzo prossimale (si confronti l'antenna di C. glacialis s. 1., maggiormente allungata, con quella di $C$. allegrettii, che è la più breve). In Ghidinia si osserva pure un allungamento/snellimento generale di tutta l'antenna, in cui però l'ultimo antennomero, pure a lati sinuati, non raggiunge mai la somma dei due precedenti (Fig. 21), che sono pure notevolmente allungati, analogamente a quanto avviene nel genere Insubriella (Fig. 22). In tutti gli altri generi trattati, per confronto, si possono trarre le medesime considerazioni (vedi oltre nelle singole descrizioni).

Metafemori. In un solo gruppo della «serie filetica di Dellabeffaella» si hanno i metafemori dentati al margine posteriore e questo, unitamente ad altri caratteri peculiari, permette di isolare il genere Stoppaniola 


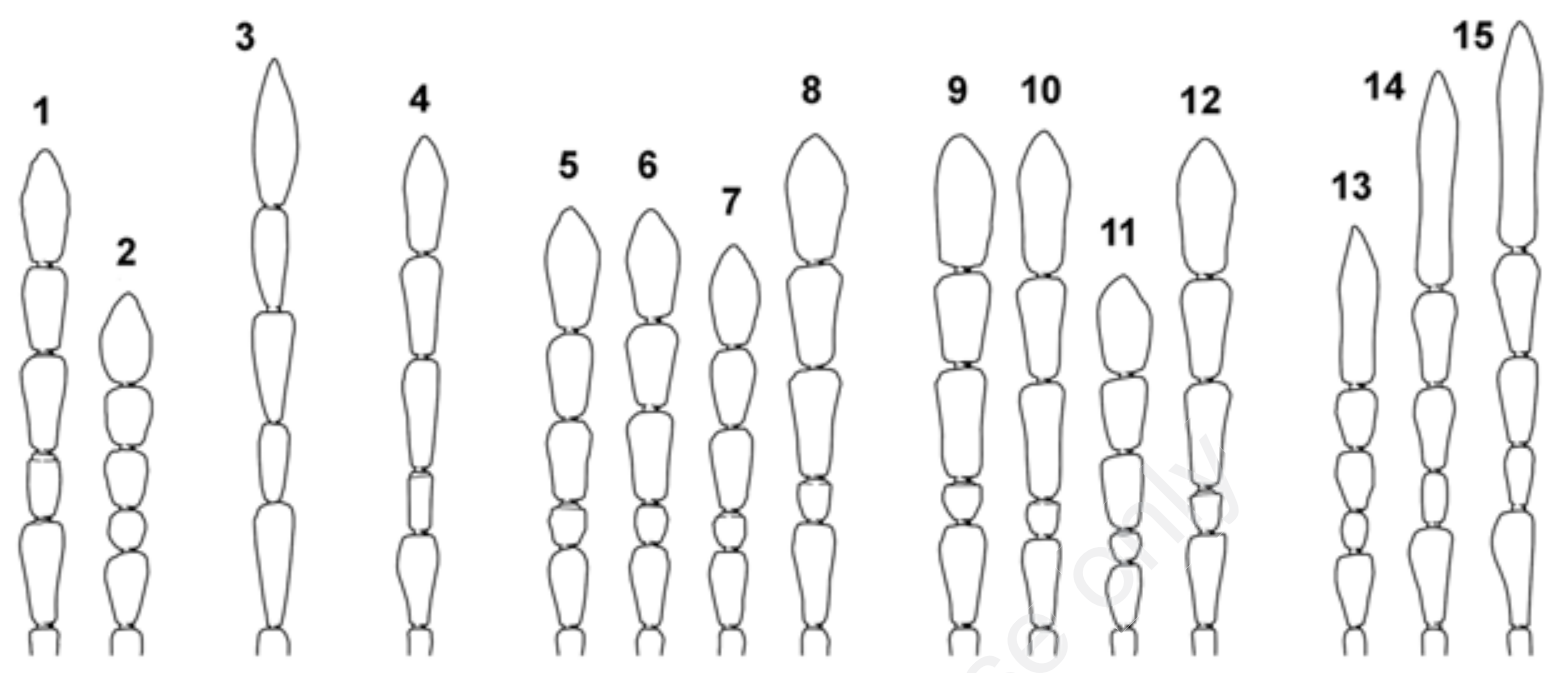

Dellabeffaella

Archeoboldoria

Canavesiella
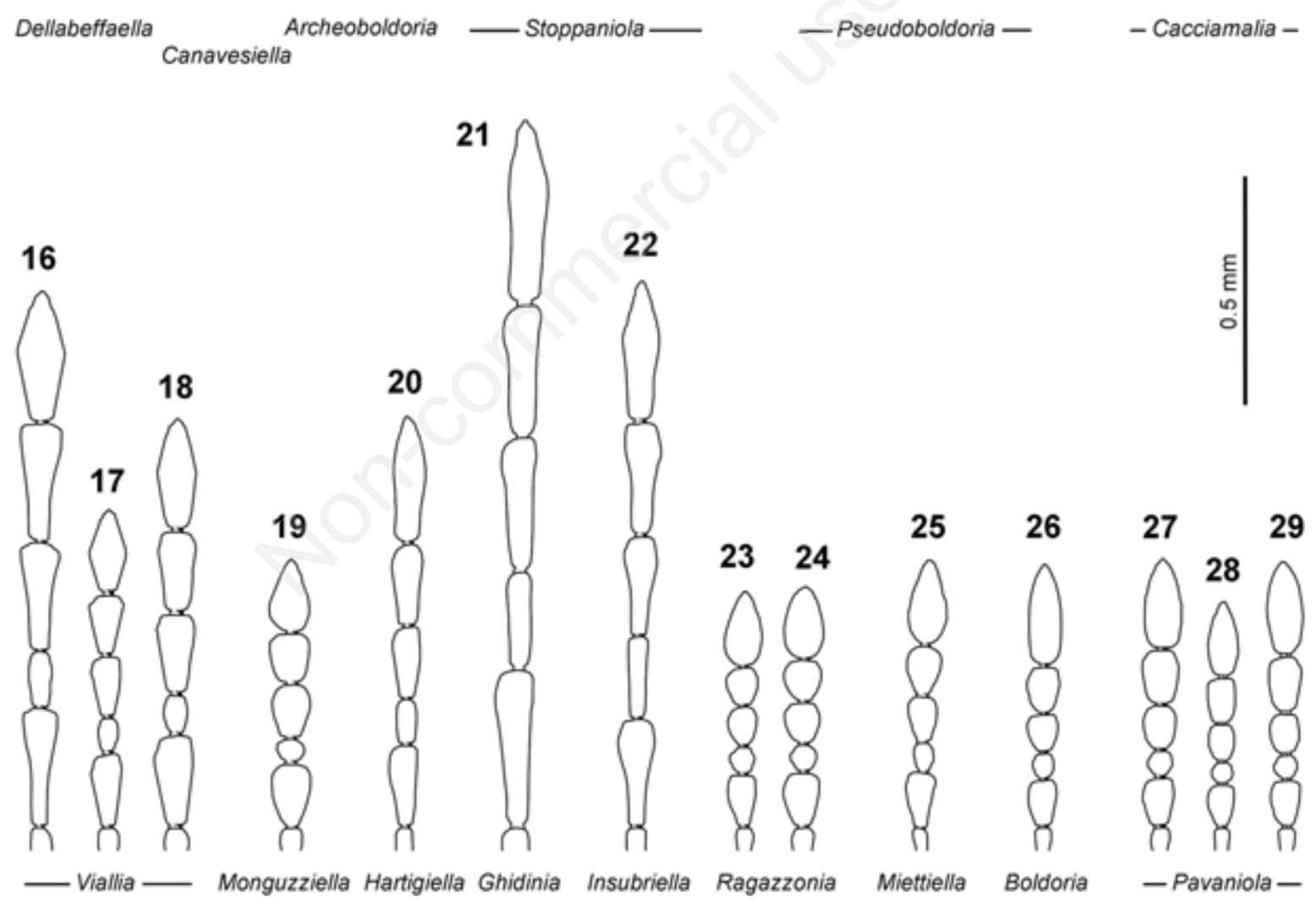

Figg. 1-29. Modelli morfologici degli ultimi cinque antennomeri (clava) maschili di alcune specie dei generi trattati. 1: Dellafeffaella roccae; 2: D. olmii; 3: Canavesiella lanai; 4: Archeoboldoria doderona; 5: Stoppaniola robiati; 6: S. malanchinii; 7 : S. bucciarellii; 8: S. comottiana; 9: Pseudoboldoria barii; 10: P. bergamasca s. str.; 11: P. kruegeri s. str.; 12: P. gratiae; 13: Cacciamalia allegrettii; 14: C. viallii; 15: C. glacialis s. str.; 16: Viallia alfanoi; 17: V. mismae; 18: V. cappai; 19: Monguzziella grottoloi; 20: Hartigiella baldensis; 21: Ghidinia aguinensis; 22: Insubriella paradoxa; 23: Ragazzonia vestae s. str.; $24: R$. trumplina; 25: Miettiella vespertilio; 26: Boldoria aculeata; 27: Pavaniola regalini; 28: P. ghidinii s. str.; 29: P. comottii. 
n. gen. dal genere Pseudoboldoria, che presenta i femori inermi, unitamente ad altri caratteri, in special modo quelli dell'edeago, ad esso peculiari.

Protarsi maschili. Il loro sviluppo in lunghezza e soprattutto la loro dilatazione, che può essere assente, moderata o spinta fino a superare in larghezza la sommità della protibia, ben caratterizzano i singoli generi.

Edeago. L'insieme dei caratteri dell'edeago, dalla morfologia del lobo mediano (forma, curvatura, apice), all'armatura setale dell'apice dei parameri, alle strutture sclerificate dell'endofallo, queste ultime a volte assenti o atrofiche, a volte ben sviluppate e complesse, si possono intravvedere profonde differenze fra i generi considerati, mentre all'interno di ogni genere si possono cogliere le differenze che individuano le singole specie come variazioni sullo stesso tema. Le immagini di edeagi riportate nel presente contributo (Figg. 30-57) riguardano solo alcune specie, a titolo di esempio, per ogni genere trattato; quando non diversamente specificato, sono tratte da Vailati (1988).

Genitali femminili. La spermateca, a volte di scarso valore specifico all'interno dei singoli generi in quanto molto simile tra specie e specie, presenta però una morfologia generale spesso caratterizzante $\mathrm{i}$ singoli generi.

Più complessa, e meno informativa, risulta la morfologia dello spiculum al margine anteriore dell'ottavo ventrite nella femmina, carattere in precedenza non preso in considerazione (Figg. 58-79). Si può presentare più o meno sviluppato in lunghezza, sottile o tozzo, con l'apice appuntito, arrotondato, quadrato o bilobato e può anche essere del tutto assente, in tal caso il margine anteriore del ventrite risulta perfettamente rettilineo. Lo scarso valore informativo è dovuto al fatto che mentre in alcuni casi sembra esistere all'interno dello stesso genere una certa omogeneità, in altri casi questa non esiste. Ad esempio, le specie del genere Cacciamalia presentano tutte lo spiculum piccolissimo, quasi scomparso, ridotto ad un ispessimento calloso (Figg. 66 e 67) e nel genere Pavaniola l'apice è bilobato (Figg. $78 \mathrm{e}$ 79). Ma nel genere Stoppaniola possiamo avere sia specie completamente prive di spiculum (Figg. 61 e 62) accanto ad altre in cui è presente, sia pure con una forma tozza e poco sviluppata (Fig. 63). Il fatto poi che la morfologia e lo sviluppo dello spiculum si mostrino così eterogenei, non solo fra i generi ma anche all'interno di uno stesso genere, non permette nemmeno di interpretarli come caratteri di specializzazione: possiamo avere una notevole riduzione nel genere Cacciamalia, possiamo avere assenza totale di spiculum in Monguzziella (Fig. 69) e in alcune specie, non tutte, di Stoppaniola (Figg. 61-63), ma possiamo avere un normale sviluppo in generi come Ghidinia (Figg. 71 e 72) o Insubriella (Fig. 73).

Anche i caratteri dei gonostili sono molto variabili tra le diverse specie. Comunque, a livello generico, salvo una più approfondita analisi di tutte le specie note, sembrerebbe diversificarsi la posizione reciproca e la distanza dall'apice del gruppo delle tre setole preapicali (Figg. 80-93).

I termini geografici, riportati nella definizione degli areali dei singoli generi e riferiti alle aree orografiche alpine e prealpine, sono usati in accordo con la SOIUSA (Marazzi, 2005).

\section{TASSONOMIA}

\section{Chiave dei generi}

1. Apice delle tibie munito al lato interno di speroni pettinati, cioè muniti di due file contrapposte di brevi denti numerosi e subeguali; cestelli apicali alle meso e metatibie incompleti; carena mesosternale, con profilo anteriore non prominente, spesso sfuggente, munita inferiormente all'angolo anteriore di un dente più $\mathrm{o}$ meno marcato, o ridotta $\mathrm{e}$ poco saliente, non prolungata posteriormente sul metasterno; metendosternite non scavato a $\mathrm{V}$, con le braccia della furca triangolari, larghe alla base («serie filetica di Dellabeffaella»)......... 2

- Apice delle tibie munito al lato interno di speroni polidentati, con un basso numero variabile di denti diseguali, mai pettinati; tibie mediane e posteriori con cestelli apicali di spine completi; carena mesosternale generalmente alta e anteriormente prominente, arrotondata, mai dentata inferiormente all'angolo anteriore, munita di apofisi posteriore libera, prolungata sul metasterno, spesso fino a superarlo; metendosternite conformato a $\mathrm{V}$, con le braccia della furca libere («serie filetica di Boldoria») . . . . . 6

2. Protarsi maschili dilatati ............. 3

- Protarsi maschili non dilatati............4

3. Protarsi maschili molto dilatati, larghi come, o anche più larghi, dell'apice della protibia; elitre provviste di una stria suturale completa e con striole trasversali fini e serrate, ma ben evidenti ............. Dellabeffaella Capra, 1924 


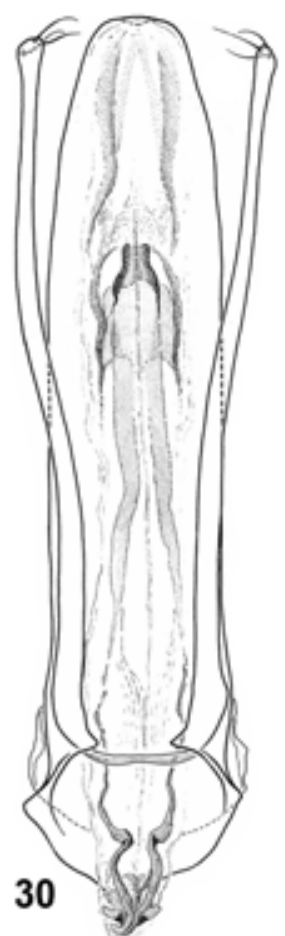

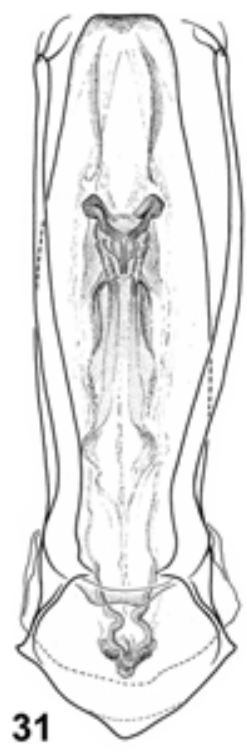
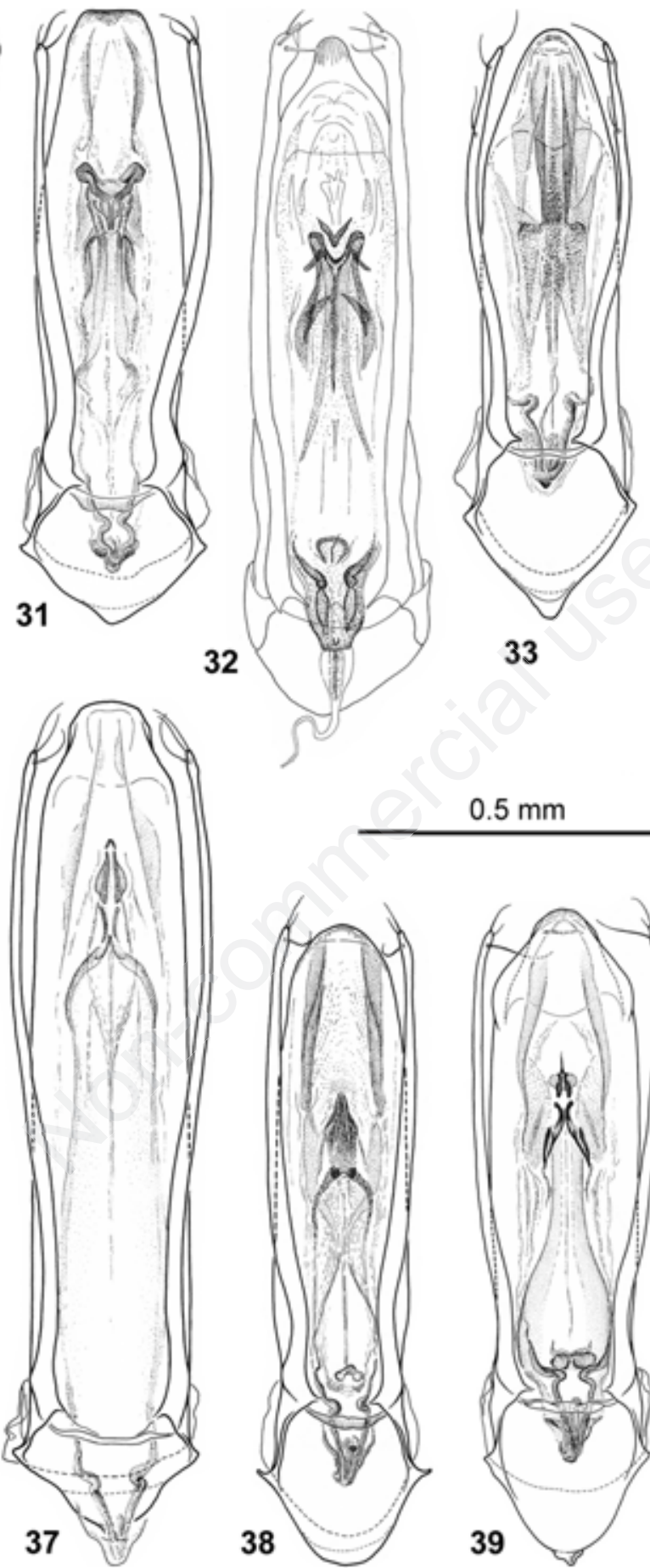

33
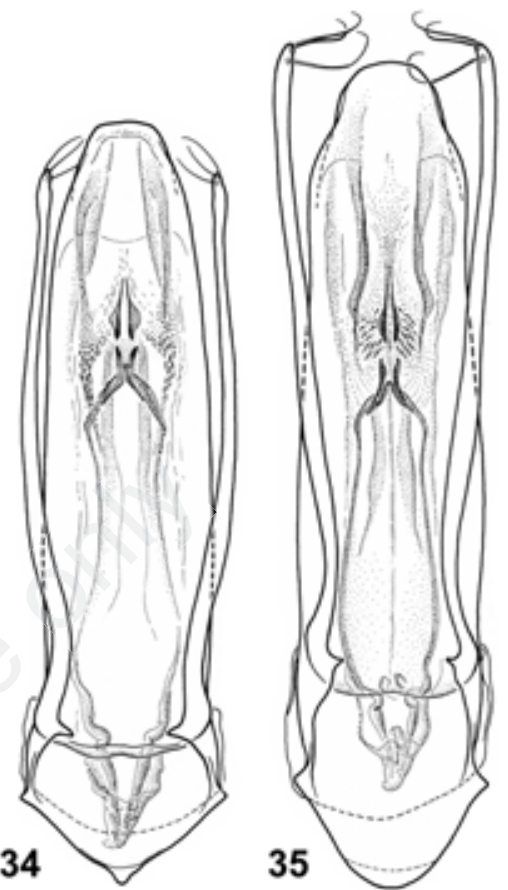

\section{$0.5 \mathrm{~mm}$}
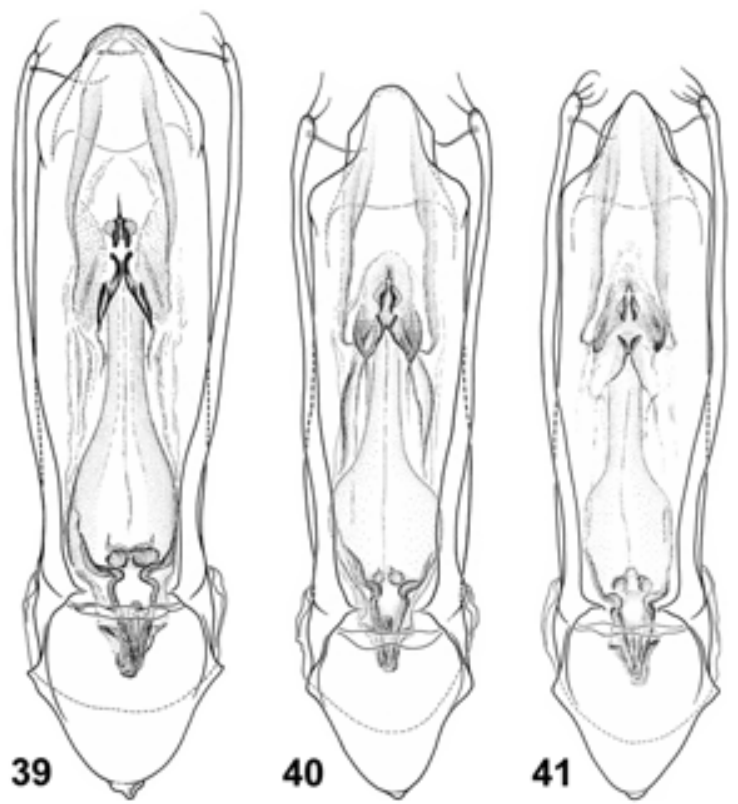

Figg. 30-41. Edeago, in visione dorsale, di alcune specie dei generi trattati. 30: Dellafeffaella roccae; 31: D. olmii; 32: Canavesiella lanai (da: Giachino, 1993); 33: Archeoboldoria doderona; 34: Stoppaniola robiati; 35: S. personata; 36: S. bucciarellii; 37: S. schatzmayri; 38: Pseudoboldoria barii; 39: P. bergamasca s. str.; 40: P. gratiae; 41: P. belluccii. 
- Protarsi maschili moderatamente dilatati, comunque più stretti dell'apice della protibia; elitre prive di stria suturale e con striole trasversali grossolane, irregolari ed evanescenti, tali da conferire loro un aspetto rasposo . . Canavesiella Giachino, 1993

4. Elitre provviste di stria suturale completa; lobo mediano dell'edeago con apice regolarmente lanceolato, appuntito; parameri generalmente con due setole apicali (in un caso con una terza preapicale) e una, ventrale, inserita lontana dall'apice. . . . . . . . . . . . . . . Archeoboldoria Ghidini, 1937

- Elitre prive di stria suturale completa, al massimo appena accennata in alcune specie per breve tratto nella regione scutellare; lobo mediano dell'edeago con apice più o meno tronco, arrotondato, non appuntito, spesso munito di espansioni laterali preapicali; parameri con tutte le setole prossime alla regione apicale..............5

5. Margine posteriore dei metafemori dentato, con piccolo dente, più o meno pronunciato, largamente ottuso, talvolta quasi svanito, ma sempre presente; lobo mediano dell'edeago non bruscamente ristretto all'apice, che appare più arrotondato, senza espansioni laterali preapicali salienti; in visione laterale presenta una curvatura più o meno accentuata; parameri muniti nella regione apicale di tre lunghe setole subeguali .... Stoppaniola n. gen.

a. edeago slanciato, allungato, con apice munito lateralmente di piccole espansioni appiattite, che gli conferiscono un aspetto spatoliforme (Figg. 35-37); in visione laterale è molto arcuato, con il profilo ventrale totalmente incavato fino alla lama basale del tegmen, senza alcuna gibbosità verso la base; fanera mediana dell'endofallo posizionata verso l'apice e senza strutture spinose o raspose ai lati . . . . . . . . ............. gruppo "schatzmayri"

b. edeago più breve e tozzo, con l'apice più arrotondato (Fig. 34); in visione laterale è meno arcuato e con evidente gibbosità basale al lato ventrale, appena avanti la lama basale del tegmen; fanera mediana dell'endofallo in posizione più o meno centrale, non spostata verso l'apice e circondata da strutture dentate o almeno raspose. . . . . . . . . gruppo "robiati"

- Margine posteriore dei metafemori liscio, inerme; lobo mediano dell'edeago più o meno ristretto all'apice, da arrotondato ad appuntito, preceduto (salvo che nella $P$. barii) da espansioni laterali preapicali a volte molto pronunciate, angolose, spianate e salienti; parameri generalmente muniti di due setole propriamente apicali e diseguali e di una preapicale più lunga $\ldots \ldots \ldots \ldots \ldots \ldots$

. . . . . . . . . Pseudoboldoria Ghidini, 1937

a. edeago di forma ogivale nella regione apicale, con apice arrotondato, senza espansioni laterali preapicali salienti (Fig. 38). . gruppo "barii"

b. edeago più tozzo, con espansioni laterali preapicali più o meno pronunciate, ma sempre arrotondate, non angolose, seguite dal restringimento apicale con l'apice più breve, meno pronunciato e più arrotondato (Fig. 39) gruppo "bergamasca"

c. edeago più slanciato, con espansioni laterali preapicali alquanto pronunciate, angolose e l'apice, oltre a queste, prolungato e ristretto (Figg. 40 e 41) ........ . gruppo "gratiae"

6. Sacco interno dell'edeago con pezzo ad $\mathrm{Y}$ basale atrofico o poco sclerificato e privo di fanere sclerificate mediane, al massimo con fasci setoso-spinulosi . . . . . . . . . . . . . . 7 Sacco interno dell'edeago sempre munito di pezzo ad Y basale sclerificato e di fanere complesse almeno nella parte mediana ... . . . . . . . 9

7. Forma generale del corpo sempre batiscioide, ovalare, con massima larghezza del pronoto alla base; antenne (Figg. 13-15) con ultimo articolo estremamente allungato, con i lati sinuati, ristretti al terzo basale e ben più lungo della somma dei due precedenti e anche pari alla somma dei tre precedenti, VIII articolo sempre allungato; lobo mediano dell'edeago munito all'apice di una linguetta più o meno pronunciata e asimmetrica, peculiarmente troncata obliquamente e rivolta verso l'alto a "becco d'oca" .......... Cacciamalia n. gen.

- Forma del corpo da batiscioide a foleuonoide; ultimo articolo delle antenne lanceolato, di poco più lungo del prededente; apice del lobo mediano dell'edeago simmetrico e rivolto verso il basso; parameri caratterizzati da due setole apicali di uguale lunghezza e di una preapicale ventrale normalmente più lunga $\ldots \ldots \ldots \ldots \ldots \ldots \ldots$

8. Forma del corpo da batiscioide a foleuonoide; antenne (Figg. 16-18) con ultimo articolo lanceolato, di forma vagamente romboidale e di poco più lungo del precedente e VIII articolo sempre più lungo che largo; nelle forme batiscioidi la carena mesosternale è alta, anteriormente prominente e 

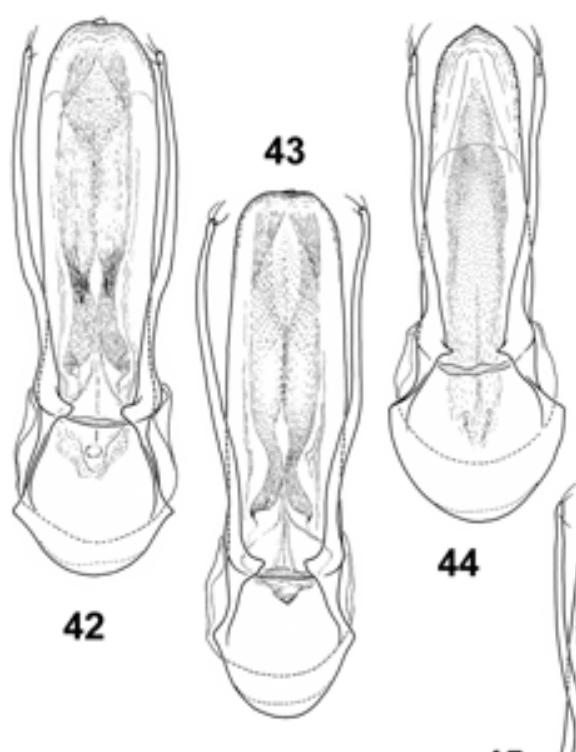

45

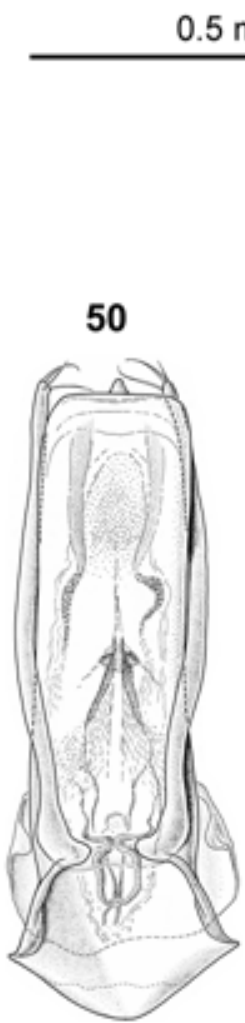

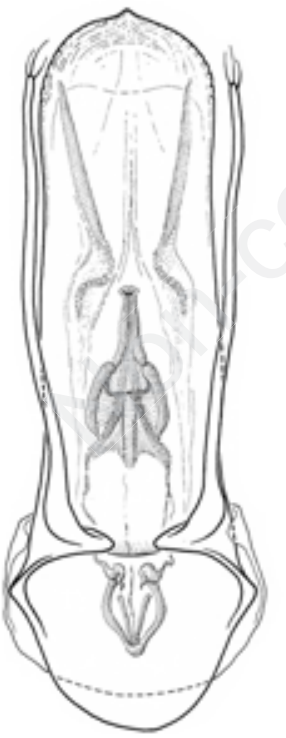

51

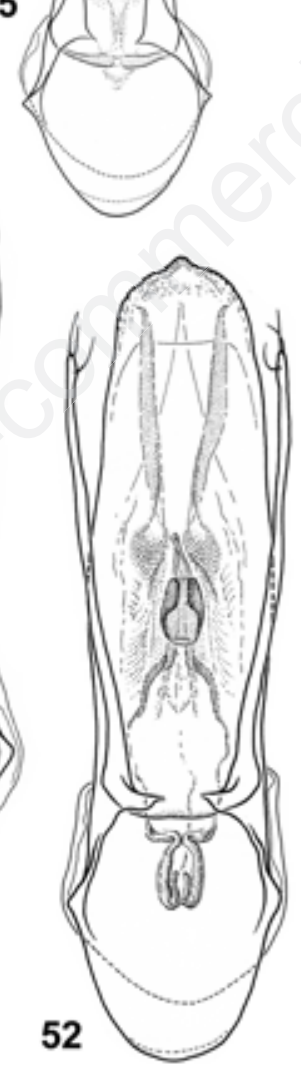

46
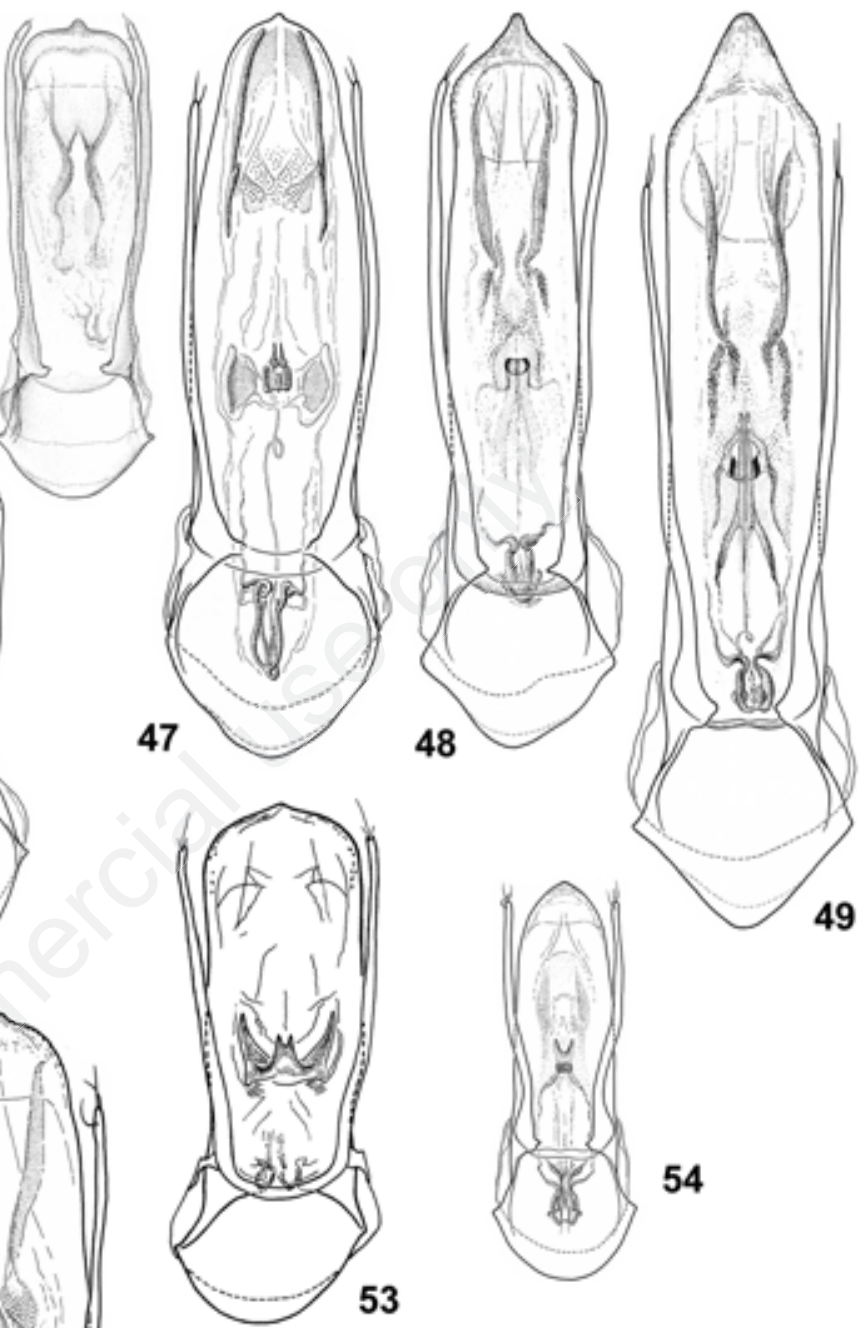

54

53
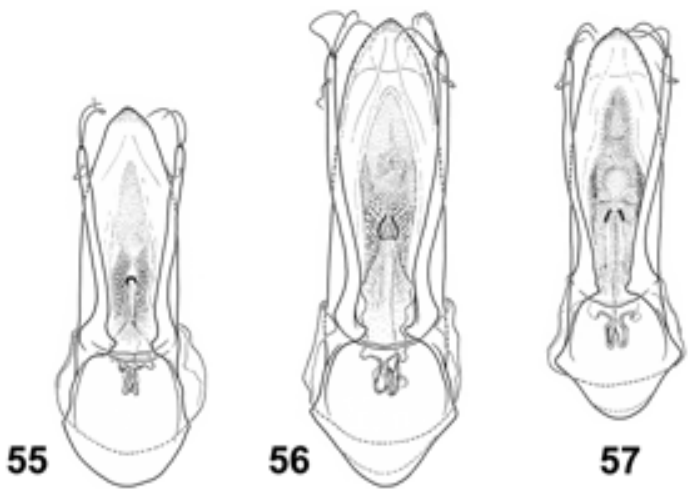

Figg. 42-57. Edeago, in visione dorsale, di alcune specie dei generi trattati. 42: Cacciamalia viallii; 43: C. glacialis 44: Viallia alfanoi; 45: V. mismae; 46: Monguzziella grottoloi; 47: Hartigiella baldensis; 48: Ghidinia aguinensis; 49: G. morettii s. str.; 50: Insubriella paradoxa; 51: Ragazzonia vestae s. str.; 52: R. trumplina; 53: Miettiella vespertilio (da: Piva, 2016); 54: Boldoria aculeata; 55: Pavaniola regalini; 56: P. longitarsis; 57: P. ghidinii s. str. 
ampiamente arrotondata, con il profilo ventrale posteriormente appiattito e allargato in forma di losanga e con l'apofisi posteriore libera prolungata ben oltre il metasterno; lobo mediano dell'edeago con l'apice ogivale, privo di linguetta apicale; in visione laterale appare con la metà distale bisinuata, assottigliata e con l'apice curvato ventralmente .............. Viallia Pavan, 1950

a. corpo a morfologia foleuonoide, con il pronoto molto più stretto delle elitre; appendici lunghe e gracili, con le antenne raggiungenti il quinto posteriore delle elitre; carena mesosternale bassa e sfuggente ....... gruppo "alfanoi"

b. corpo a morfologia batiscioide, globoso, con il pronoto largo alla base quanto le elitre; antenne raggiungenti al massimo il terzo posteriore delle elitre; carena mesosternale alta, anteriormente prominente e ampiamente arrotondata .................. gruppo "mismae"

- Forma generale del corpo batiscioide, ma allungata, con avancorpo ristretto, con la massima larghezza del pronoto avanti la base, che è ristretta; antenne (Fig. 19) con ultimo articolo piriforme, di poco più lungo del precedente e VIII decisamente trasverso; carena mesosternale particolarmente bassa, con il profilo anteriore arrotondato ma sfuggente e con l'apofisi posteriore libera prolungata solo parzialmente sul metasterno; lobo mediano dell'edeago con l'apice largo e tronco, non ogivato, munito di linguetta apicale pronunciata; in visione laterale si presenta robusto e regolarmente arcuato .......... Monguzziella Vailati, 1993

9. Specie di taglia media o grande (lunghezza 1.78$3.34 \mathrm{~mm}$ ); protarsi maschili sensibilmente dilatati, più larghi o larghi quanto la sommità della protibia; sacco interno dell'edeago con due evidenti liste sclerificate subparallele nella regione apicale . . 10

- Specie di taglia più piccola (lunghezza 1.45-1.95 $\mathrm{mm}$ ); protarsi maschili pochissimo dilatati, ben più stretti dell'apice della protibia; sacco interno dell'edeago senza liste sclerificate apicali, ma solo con fanere mediane che si prolungano distalmente con fasci setoso-spinulosi . . . . . . . . . 14

10. Protarsi maschili, oltre che molto dilatati, particolarmente sviluppati in lunghezza, lunghi circa quanto, $\mathrm{o}$ anche più, della protibia. ..........

Hartigiella Müller, 1935

- Protarsi maschili ben più brevi della protibia ...
11. Corpo grande e slanciato (lunghezza 2.74-3.34 $\mathrm{mm}$ ); pronoto campaniforme, con i lati da debolmente a visibilmente sinuati presso la base; antenne allungate, filiformi, raggiungenti circa l'apice delle elitre, con l'VIII articolo da poco meno a più di quattro volte più lungo che largo; edeago in visione laterale con l'apice diritto o lievemente rivolto verso l'alto........... 12

- Corpo più piccolo, tozzo e globoso (lunghezza $1.78-2.34 \mathrm{~mm}$ ), con i lati del pronoto regolarmente arrotondati fino alla base; antenne brevi, raggiungenti al massimo il terzo basale delle elitre, con l'VIII articolo da trasverso a lungo al massimo una volta e mezza la sua larghezza e l'XI appena lanceolato o piriforme; edeago in visione laterale con l'apice rivolto verso il basso; sacco interno dello stesso con grande fanera mediana sclerificata e complessa..................... 13

12. Primi due antennomeri, anche se in rapporto lievemente variabile, tendenzialmente di uguale lunghezza; pronoto debolmente sinuato ai lati; carena mesosternale alta, con profilo anteriore fortemente arrotondato e prominente e con l'apofisi posteriore prolungata a oltrepassare il margine posteriore del metasterno .......... Ghidinia Pavan, 1939

- Primo antennomero ben più lungo del secondo; pronoto marcatamente ristretto e sinuato ai lati tra il terzo e il quarto basale; carena mesosternale bassa, con il profilo anteriore non prominente, obliquamente sfuggente e con l'apofisi posteriore prolungata sul metasterno fin quasi a raggiungerne il margine posteriore, ma senza superarlo ...... ............... Insubriella Vailati, 1990

13. Ottavo antennomero da trasverso a lungo al massimo quanto largo; carena mesosternale con apofisi posteriore libera prolungata sul metasterno fino a superarlo; sacco interno dell'edeago con pezzo basale ad $\mathrm{Y}$ ben sviluppato e complesso e grande fanera mediana sclerificata di forma complessa (Figg. 51 e 52)......... Ragazzonia $\mathrm{n}$. gen.

- Ottavo antennomero lungo una volta e mezza la larghezza; carena mesosternale con apofisi posteriore libera prolungata solo per breve tratto sul metasterno; sacco interno dell'edeago con pezzo basale ad $\mathrm{Y}$ poco sviluppato e poco definito e fanera mediana sclerificata riproducente la sagoma di un chirottero (Fig. 53) ......... Miettiella Piva, 2016

14. Antenne con ultimo articolo allungato, lungo come la somma dei due precedenti più metà dell'VIII 


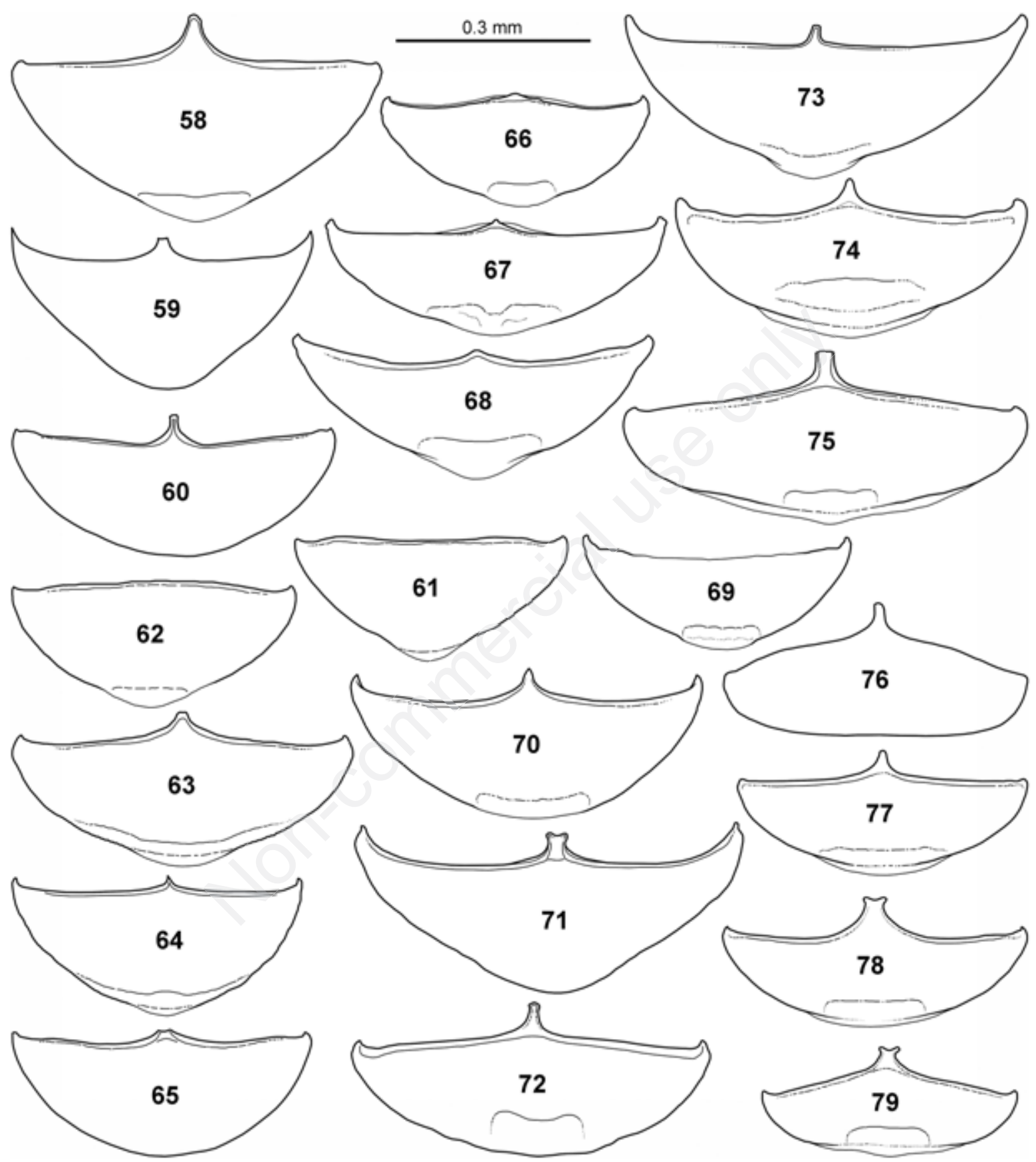

Figg. 58-79. VIII ventrite della femmina di alcune specie dei generi trattati. 58: Dellafeffaella olmii; 59: Canavesiella lanai (da: Giachino, 1993); 60: Archeoboldoria doderona; 61: Stoppaniola malanchinii; 62: S. focarilei; 63: S. schatzmayri; 64: Pseudoboldoria bergamasca s. str.; 65: P. gratiae; 66: Cacciamalia allegrettii; 67: C. glacialis s. str.; 68: Viallia cappai; 69: Monguzziella grottoloi; 70: Hartigiella baldensis; 71: Ghidinia morettii s. str.; 72: G. vailatii; 73: Insubriella paradoxa; 74: Ragazzonia vestae s. str.; 75: R. trumplina; 76: Miettiella vespertilio (da: Piva, 2016); 77: Boldoria aculeata; 78: Pavaniola longitarsis; 79: P. ghidinii s. str. 
(Fig. 26); parameri dell'edeago muniti all'apice di tre setole di normale morfologia e lunghezza ... . . . . . . . . . . . Boldoria Jeannel, 1924

- Antenne (Figg. 27-29) con ultimo articolo generalmente più breve $\mathrm{o}$ al massimo pari alla somma dei due precedenti ; parameri dell'edeago muniti di norma di quattro setole, in un caso (B. regalini) di tre, ma sempre molto lunghe, flagelliformi (Figg. 55-57) ........... Pavaniola $\mathrm{n}$. gen.

a. parameri dell'edeago muniti all'apice di quattro setole, di cui tre propriamente apicali e una preapicale, leggermente arretrata prossimalmente; corpo più o meno convesso e di forma ellittica. ............ . gruppo "ghidinii"

b. parameri dell' edeago con tutte quattro le setole raccolte all'apice; corpo appiattito, allungato e a lati paralleli ........ gruppo "comottii"

c. parameri dell'edeago muniti all'apice di tre sole setole, di cui due propriamente apicali e una preapicale, leggermente arretrata prossimalmente; corpo leggermente ovoidale, con il pronoto a base larga e le elitre attenuate in addietro .............. gruppo "regalini"

\section{Serie filetica di «Dellabeffaella»}

genere Dellabeffaella Capra, 1924

(Figg. 1, 2, 30, 31, 58)

Royerella (Della Beffaella) Capra, 1924, Atti reg. Acc. Sci. Torino: LIX: 155.

Dellabeffaella Capra: Vailati, 1988: 28.

Dellabeffaella Capra: Newton, 1998: 115.

Dellabeffaella Capra: Perreau, 2000: 265.

Dellabeffaella Capra: Perreau, 2004: 164.

SPECIE TIPO: Royerella (Della-Beffaella) Roccae Capra, 1924.

RIDESCRIZIONE. Corpo batiscioide, di grande taglia, di forma da ellittica a ovoidale, con avancorpo largo.

Antenne del $\widehat{\partial}$ moderatamente allungate, raggiungenti al massimo, distese all'indietro, la metà delle elitre; articolo I ben più breve del II, VIII da tanto lungo quanto largo a lungo quasi due volte la larghezza, XI lanceolato e lungo come il precedente più quasi la metà del IX (Figg. 1 e 2).

Pronoto con i lati regolarmente arrotondati, parti- colarmente ampio e globoso, largo come e anche più largo delle elitre, con la massima larghezza ben più avanti degli angoli posteriori, che sono acuti e rivolti in addietro per l'accentuata sinuosità della base che è anche sensibilmente ristretta.

Carena mesosternale alta, ma non prominente in avanti, il profilo anteriore obliquamente sfuggente e quello ventrale lievemente sinuato formano un angolo ottuso, munito inferiormente di un dente più o meno pronunciato, a volte arrotondato ed evanescente; posteriormente è priva di apofisi prolungata sul metasterno.

Elitre provviste di stria suturale completa e ben marcata e con striole trasversali fini e serrate.

Apparato metatergale grande, con apofisi posteriore lunga almeno come un quarto delle elitre.

Femori posteriori con margine posteriore normale, inerme.

Apice delle tibie munito di speroni interni pettinati, meso e metatibie con cestelli apicali incompleti di spine brevi e rade.

Protarsi maschili fortemente dilatati, larghi come, o più larghi, dell'apice della protibia.

Lobo mediano dell'edeago (Figg. 30 e 31) di forma allungata, lungo circa un terzo del corpo; in visione dorsale con i lati subparalleli nella metà basale, quindi convergenti verso l'apice che è ogivato e appuntito; in visione laterale si presenta ampiamente e regolarmente curvato, snello, con l'apice marcatamente rivolto in basso.

Sacco interno con pezzo basale ad $\mathrm{Y}$ grande e ben sclerificato e con una fanera mediana grande e complessa; sono presenti due liste apicali parallele moderatamente sclerificate.

Parameri lunghi ed esili, di poco più brevi del lobo mediano, muniti all'apice di tre setole subeguali.

Spiculum dell'ottavo ventrite nella femmina poco sviluppato in lunghezza, tozzo, triangolare, leggermente ingrossato all'estremità (Fig. 58).

Spermateca grande, con la parte prossimale globosa e la parte distale più piccola e allungata; ductus distalmente sclerificato per breve tratto.

Vi appartengono, allo stato attuale delle conoscenze, le seguenti specie:

Dellabeffaella roccae (Capra, 1924)

Dellabeffaella olmii Casale, 1980

Distribuzione nOTA. Alpi Cozie centrali e meridio- 


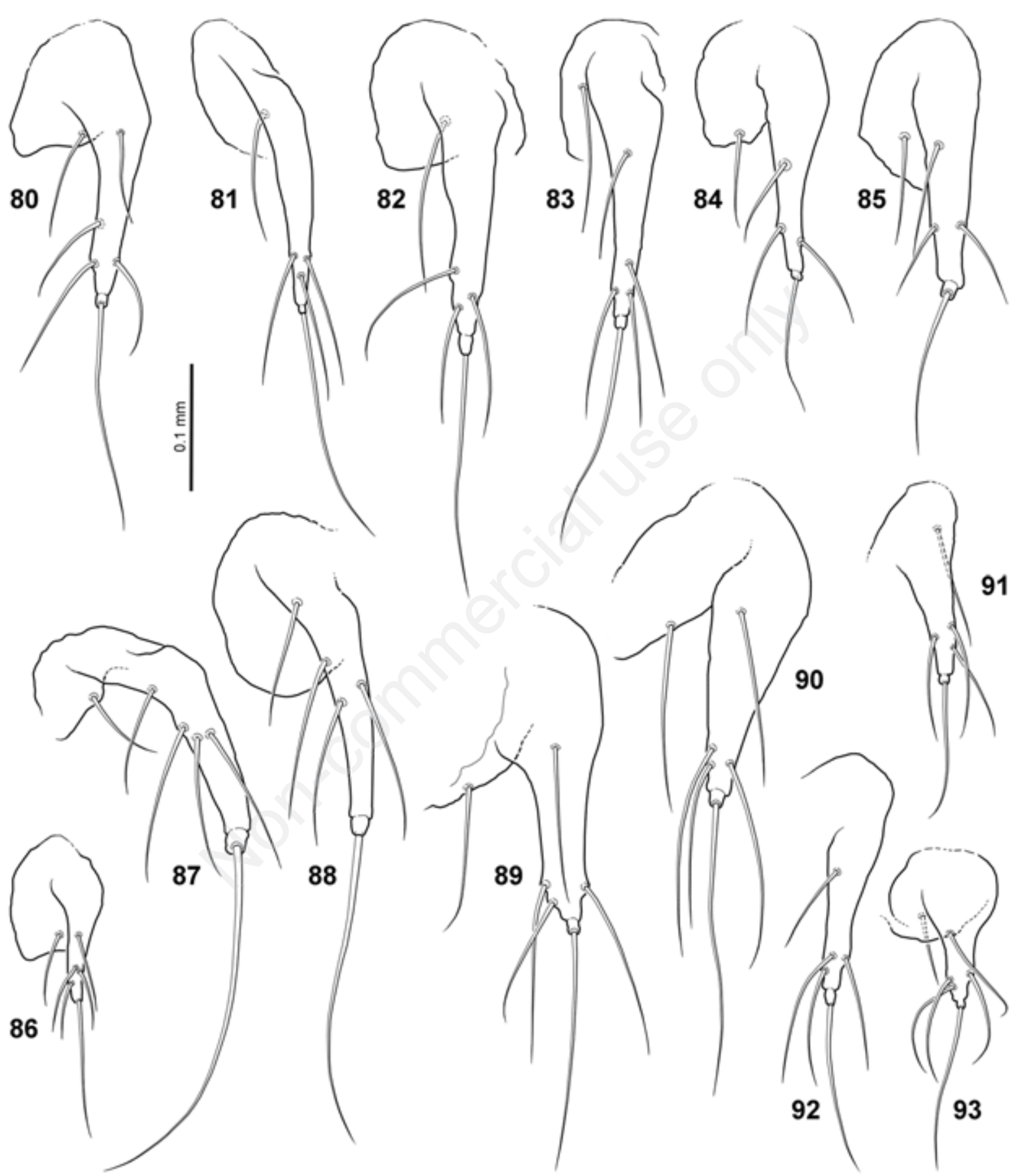

Figg. 80-93. Gonostilo della femmina di alcune specie dei generi trattati. 80: Archeoboldoria doderona; 81: Stoppaniola malanchinii; 82: S. schatzmayri; 83: Pseudoboldoria bergamasca s. str.; 84: Cacciamalia allegrettii; 85: C. glacialis s. str.; 86: Monguzziella grottoloi; 87: Ghidinia morettii s. str.; 88: G. vailatii; 89: Ragazzonia vestae s. str.; 90: R. trumplina; 91: Boldoria aculeata; 92: Pavaniola longitarsis; 93: P. ghidinii s. str. 
nali: Val Pellice e Val Chisone; Alpi Graie sud-orientali: Valle di Lanzo.

genere Canavesiella Giachino, 1993

(Figg. 3, 32, 59)

Canavesiella Giachino, 1993, Boll. Mus. reg. Sci. nat. Torino, 11 (2): 349.

Canavesiella Giachino: Newton, 1998: 114.

Canavesiella Giachino: Perreau, 2000: 264.

Canavesiella Giachino: Perreau, 2004: 163.

SPECIE TIPO: Canavesiella lanai Giachino.

RIDESCRIZIONE. Corpo batiscioide, di taglia mediogrande, di forma ellittica particolarmente allungata, con accentuato restringimento alla base del pronoto, che divide il corpo in due parti.

Antenne del $\hat{\partial}$, allungate, superanti, distese all'indietro, il terzo distale delle elitre; articolo I di poco più lungo del II, VIII allungato, più di due volte più lungo che largo, XI lanceolato e lungo come il precedente più la metà del IX (Fig. 3).

Pronoto con i lati regolarmente arrotondati, con la massima larghezza appena prima della metà e particolarmente ristretto alla base, che si presenta subrettilinea, solo debolmente arcuata presso gli angoli posteriori quasi retti, ma smussati.

Carena mesosternale bassa, estremamente ridotta, appena indicata.

Elitre prive di stria suturale e con striole trasversali grossolane, irregolari ed evanescenti, tali da conferire loro un aspetto rasposo.

Apparato metatergale piccolo, con apofisi posteriore brevissima, con la doccia longitudinale di poco più lunga della larghezza.

Femori posteriori con margine posteriore inerme.

Apice delle tibie munito di speroni interni pettinati, meso e metatibie con cestelli apicali incompleti di spine brevi e rade.

Protarsi maschili moderatamente dilatati, ben più stretti dell'apice della protibia.

Lobo mediano dell'edeago (Fig. 32) di forma allungata, lungo circa un terzo del corpo; in visione dorsale con i lati subparalleli fino alla zona preapicale, quindi convergenti verso l'apice che è munito di linguetta apicale prominente, arrotondata, non appuntita; in visione laterale si presenta ampiamente e regolar- mente curvato, snello, con l'apice appiattito e bruscamente rivolto verso l'alto.

Sacco interno con pezzo basale ad Y voluminoso e ben sclerificato e con una fanera mediana grande e complessa; sono assenti altre fanere nella regione apicale.

Parameri lunghi ed esili, di poco più lunghi del lobo mediano; apice dilatato a cucchiaio e munito di tre setole subeguali.

Spiculum dell'ottavo ventrite nella femmina piccolo, tozzo, ridotto ad una linguetta poco sviluppata in lunghezza e con l'apice tronco, sub quadrato (Fig. 59).

Spermateca grande, allungata, con la parte prossimale globosa e la parte distale più piccola e allungata; ductus distalmente sclerificato per breve tratto.

$\mathrm{Vi}$ appartengono, allo stato attuale delle conoscenze, le seguenti specie:

Canavesiella lanai Giachino, 1993

Canavesiella casalei Giachino, 1993

DistRIBUZIONE NOTA. Alpi Graie nord-orientali: Valle di Ribordone e Val Chiusella.

genere Archeoboldoria Ghidini, 1937

(Figg. 4, 33, 60, 80)

Boldoria (Archeoboldoria) Ghidini, 1937, Mem. Soc. entom. It., XVI: 69.

Archeoboldoria Ghidini: Vailati, 1988: 39.

Archeoboldoria Ghidini: Newton, 1998: 113.

Archeoboldoria Ghidini: Perreau, 2000: 264.

Archeoboldoria Ghidini: Perreau, 2004: 159.

SpeCIE TIPO: Bathysciola (Boldoria) Doderona Jeannel, 1924.

RIDESCRIZIONE. Corpo batiscioide, di media taglia, di forma allungata, leggermente ristretto alla base del pronoto.

Antenne del $\hat{\delta}$, moderatamente allungate, superanti, distese all'indietro, la metà e anche raggiungenti il terzo distale delle elitre; articolo I appena più breve del II, VIII da una volta e mezza a più di due volte più lungo che largo, XI lanceolato e di poco più lungo del precedente (Fig. 4).

Pronoto con i lati da regolarmente arrotondati a rettilinei e marcatamente convergenti nella metà ante- 
riore, con angoli posteriori da acuti a quasi retti e la base ristretta.

Carena mesosternale ottusa, con il profilo anteriore obliquamente sfuggente, poco arcuato inferiormente munita di un dente molto pronunciato ma non appuntito, dovuto alla sinuosità del profilo inferiore; posteriormente priva di apofisi prolungata sul metasterno.

Elitre provviste di stria suturale completa e ben marcata e con striole trasversali forti, ben evidenti sul disco.

Apparato metatergale con apofisi posteriore allungata e stretta, lunga almeno come un quinto delle elitre, con la doccia longitudinale sei-sette volte più lunga che larga.

Femori posteriori con margine posteriore inerme.

Apice delle tibie munito di speroni interni pettinati, meso e metatibie con cestelli apicali incompleti di spine brevi e rade.

Protarsi maschili per nulla dilatati, larghi circa come la metà dell'apice della protibia.

Lobo mediano dell'edeago (Fig. 33) tozzo, poco allungato, lungo circa un terzo del corpo, di forma ogivale nella metà distale, ma non appuntito; in visione laterale si presenta moderatamente curvato, con l'apice lievemente rivolto in basso.

Sacco interno con pezzo basale ad $\mathrm{Y}$ ben sclerificato e con una fanera mediana poco definita, circondata da fasci setoso-spinulosi che si prolungano distalmente con due liste parzialmente sclerificate.

Parameri lunghi, di poco più brevi del lobo mediano, dilatati all'apice in forma di spatola triangolare, muniti di una setola apicale, una (o due in A. pascuttoi) preapicali e una, ventrale, molto spostata prossimalmente, dal terzo distale a quasi metà paramero (ad eccezione di $A$. pascuttoi, in cui è molto più avanzata, circa al sesto distale del paramero) e posta nel punto in cui vi è un notevole cambio di spessore del paramero stesso.

Spiculum dell'ottavo ventrite nella femmina poco sviluppato e sottile (Fig. 60).

Spermateca quasi simmetrica, con le parti prossimale e distale sclerificate e poco dilatate, piccole e ugualmente allungate; ductus distalmente sclerificato per breve tratto.

Vi appartengono, allo stato attuale delle conoscenze, le seguenti specie:

Archeoboldoria doderona (Jeannel, 1924)

Archeoboldoria lanai Giachino \& Vailati, 1997
Archeoboldoria pascuttoi Giachino, Lana \& Vailati, 2001

Archeoboldoria sturanii Casale \& Giachino, 2010

DisTRIBUZIONE NOTA. Alpi Graie nord-orientali: all'imbocco della Valle d'Aosta, sul versante destro; Alpi Biellesi e Cusiane: tra la Dora Baltea e il lago d'Orta.

genere Stoppaniola $\mathrm{n}$. gen.

(Figg. 5-8, 34-37, 61-63, 81, 82)

Pseudoboldoria «gruppo robiatii»: Vailati, 1988: 49.

Pseudoboldoria «gruppo schatzmayri»: Vailati, 1988: 68.

Pseudoboldoria Ghidini (pars): Newton, 1998: 118.

Pseudoboldoria groupe «robiati»: Perreau, 2000: 267.

Pseudoboldoria groupe «schatzmayeri»: Perreau, 2000: 267.

Pseudoboldoria Ghidini (pars): Perreau, 2004: 170.

SpeCIE TIPO: Bathyscia Robiati Reitter, 1889, Ann. Mus. civ. St. nat. Genova, XXVII: 293.

DesCRIZIONE. Corpo batiscioide, di media taglia, generalmente di forma ellittica, a volte lievemente ristretto in addietro.

Antenne del $\widehat{\partial}$ moderatamente allungate, raggiungenti di norma, distese all'indietro, circa la metà delle elitre; rapporto fra la lunghezza dei primi due articoli variabile, l'articolo I può essere più breve, uguale o più lungo del II, VIII da trasverso ad appena più lungo che largo, XI da lanceolato a romboidale e lungo come il precedente più quasi la metà del IX (Figg. 5-8).

Pronoto largo alla base, con i lati sempre regolarmente arrotondati.

Carena mesosternale alta, ma non prominente in avanti, il profilo anteriore obliquamente sfuggente, più o meno leggermente curvo e quello ventrale da diritto a lievemente sinuato, sempre munita inferiormente all'angolo anteriore di un dente piccolo, ma ben pronunciato; posteriormente priva di apofisi prolungata sul metasterno.

Elitre prive di stria suturale e provviste di striole trasversali, da fini a grossolane, ma sempre ben marcate.

Apparato metatergale da mediamente sviluppato a grande, ma tozzo, con apofisi posteriore breve, con la doccia longitudinale da due a tre volte più lunga che larga. 
Femori posteriori muniti al centro del margine posteriore di un piccolo dente, piu o meno pronunciato, talvolta evanescente, ma sempre presente.

Apice delle tibie munito di speroni interni pettinati, meso e metatibie con cestelli apicali incompleti di spine brevi e rade.

Protarsi maschili mai dilatati, larghi circa la metà dell'apice della protibia.

Lobo mediano dell'edeago (Figg. 34-37) particolarmente allungato, lungo da poco meno a più della metà del corpo; in visione dorsale con i lati subparalleli per quasi tutta la lunghezza, quindi un poco ristretti verso l'apice che è arrotondato, spatoliforme; in visione laterale si presenta regolarmente curvato, snello, con l'apice diritto.

Sacco interno con pezzo basale ad Y ben sviluppato e sclerificato e con una fanera mediana, ma in posizione avanzata nella metà distale, formata da una placchetta alettata e da due braccia simmetriche "a compasso" rivolte prossimalmente e che si prolungano fino alle braccia del pezzo ad Y; sono presenti due liste apicali subparallele moderatamente sclerificate.

Parameri lunghi ed estremamente esili, di poco più brevi o lunghi quanto il lobo mediano, muniti all'apice di tre setole.

Ottavo ventrite nella femmina privo di spiculum in alcune specie, in altre con il bordo anteriore al massimo appena segnato da un angolo ampiamente ottuso o con spiculum appena pronunciato, tozzo, triangolare (Figg. 61-63).

Spermateca con la parte prossimale poco allungata e più grande della parte distale che è sferica e meno voluminosa; parte mediana generalmente allungata, almeno il doppio della larghezza.

Vi appartengono, allo stato attuale delle conoscenze, le seguenti specie:

Gruppo «robiati» Vailati, 1988

Stoppaniola robiati (Reitter, 1889)

Stoppaniola personata (Vailati, 1988)

Stoppaniola focarilei (Vailati, 1988)

Stoppaniola malanchinii (Pavan \& Ronchetti, 1949)

Gruppo «schatzmayri» Vailati, 1988

Stoppaniola bucciarellii (Rossi, 1963)

Stoppaniola schatzmayri (Focarile, 1952)

Stoppaniola comottiana (Vailati, 1988)

DERIVATIO NOMINIS. Questo nuovo genere è dedicato, anche in ragione dell'area geografica nella quale è distribuito, alla memoria dell'abate Antonio Stoppani di Lecco (1824-1891), straordinario geologo, paleontologo, alpinista e appassionato esploratore delle Prealpi lombarde, universalmente noto per la sua opera "Il Bel Paese".

DistribuZione nOta. Prealpi Comasche: massiccio del M. Generoso e Triangolo Lariano, oltre a una stazione a sud dell'anfiteatro morenico del lago di Como (ramo di Lecco), praticamente già in pianura Padana, insediata negli affioramenti conglomeratici del "Ceppo d'Adda"; Prealpi Bergamasche: Gruppo del M. Resegone, Valle Imagna, alta Valsassina, monti tra le Valli Brembana e Seriana e colline pedemontane tra la bassa Valle Brembana e la Val Cavallina.

genere Pseudoboldoria Ghidini, 1937

(Figg. 9-12, 38-41, 64, 65, 83)

Pseudoboldoria «gruppo barii» Vailati, 1988: 83.

Pseudoboldoria «gruppo bergamasca» Vailati, 1988: 88. Pseudoboldoria «gruppo gratiae» Vailati, 1988: 117. Pseudoboldoria Ghidini (pars): Newton, 1998: 118. Pseudoboldoria groupe «barii»: Perreau, 2000: 265. Pseudoboldoria groupe «bergamasca »: Perreau, 2000: 265.

Pseudoboldoria groupe «gratiae »: Perreau, 2000: 266. Pseudoboldoria Ghidini (pars): Perreau, 2004: 170.

SPECIE TIPO: Bathyscia (Bathysciola) Krügeri Müller, 1914, Sb. math. naturw. Kl. Akad. Wiss., 123: 1013.

RIDESCRIZIONE. Corpo batiscioide, di media taglia, generalmente di forma ellittica.

Antenne del ồ moderatamente allungate, raggiungenti di norma, distese all'indietro, circa la metà delle elitre; articolo I di lunghezza uguale o più breve del II, VIII generalmente trasverso o appena piu lungo che largo, XI tozzo, largo, appiattito, arrotondato all'apice, lungo al massimo come il precedente più un terzo del IX (Figg. 9-12).

Pronoto largo alla base, con i lati sempre regolarmente arrotondati.

Carena mesosternale alta, ma non prominente in avanti, il profilo anteriore da obliquamente sfuggente a retto, più o meno leggermente curvo e quello ventrale diritto, sempre munita inferiormente all'angolo ante- 
riore di un dente piccolo, ma ben pronunciato; posteriormente priva di apofisi prolungata sul metasterno.

Elitre prive di stria suturale, ma a volte presente con una debolissima traccia al quarto basale nella regione scutellare che si presenta depressa, provviste di striole trasversali, da fini a grossolane, da leggere a ben marcate.

Apparato metatergale mediamente sviluppato, tozzo, con apofisi posteriore breve, con la doccia longitudinale da due a tre volte più lunga che larga.

Femori posteriori normali, con il margine posteriore inerme.

Apice delle tibie munito di speroni interni pettinati, meso e metatibie con cestelli apicali incompleti di spine brevi e rade.

Protarsi maschili mai dilatati, larghi anche meno della metà dell'apice della protibia.

Lobo mediano dell'edeago (Figg. 38-41) più tozzo che in Stoppaniola, in genere lungo come un terzo della lunghezza del corpo; in visione dorsale con i lati subparalleli per quasi tutta la lunghezza, quindi largo nella regione preapicale, appiattito con espansioni dorso-laterali più o meno pronunciate, spianate e sempre salienti; in visione laterale si presenta moderatamente curvato, con l'apice diritto.

Sacco interno con pezzo basale ad Y ben sviluppato e sclerificato, con una fanera complessa in posizione mediana e due liste apicali subparallele moderatamente sclerificate.

Parameri lunghi ma non esili, di poco più brevi del lobo mediano, di norma muniti all'apice di due setole diseguali e di una terza preapicale ventrale più lunga e robusta.

Spiculum dell'ottavo ventrite nella femmina presente, ma piccolissimo, appuntito o appena accennato da una protuberanza incavata (Figg. 64 and 65).

Spermateca tendenzialmente simmetrica, con le parti prossimale e distale della stessa dimensione; parte mediana generalmente breve, lunga meno del doppio della larghezza.

Vi appartengono, allo stato attuale delle conoscenze, le seguenti specie:

Gruppo «barii» Vailati, 1988

Pseudoboldoria barii Focarile, 1950

Gruppo «bergamasca» Vailati, 1988

Pseudoboldoria bergamasca bergamasca (Jeannel, 1914)
Pseudoboldoria bergamasca binaghii Jeannel, 1931

Pseudoboldoria kruegeri kruegeri (Müller, 1914)

Pseudoboldoria kruegeri orobica Vailati, 1988

Pseudoboldoria intermedia Vailati, 1988

Pseudoboldoria longobarda Vailati, 1988

Gruppo «gratiae» Vailati, 1988

Pseudoboldoria gratiae Monguzzi, 1984

Pseudoboldoria belluccii Regalin, 1985

DistribuZione nota. Alpi Orobie e Prealpi Bergamasche: massiccio delle Grigne, M. Resegone, Valle Imagna, Valli Brembana e Seriana, Val Cavallina, Sebino bergamasco e fino alla media Val Camonica; Prealpi Bresciane: M. Guglielmo e versante destro della Val Trompia.

\section{Serie filetica di «Boldoria»}

genere Cacciamalia n. gen.

(Figg. 13-15, 42, 43, 66, 67, 84, 85)

Boldoria «gruppo glacialis»: Vailati, 1988: 157.

Boldoria Jeannel (pars): Newton, 1998: 114.

Boldoria groupe «glacialis»: Perreau, 2000: 252.

Boldoria Jeannel (pars): Perreau, 2004: 162.

SPECIE TIPO: Boldoria (s. str.) glacialis Vailati, 1975, Natura Bresciana, 11: 26.

DesCrizIONE. Corpo batiscioide di media taglia, ellittico allungato, più o meno attenuato posteriormente.

Antenne del $\widehat{o}$ esili e particolarmente allungate, distese all'indietro, raggiungenti dalla metà al quarto distale delle elitre; articolo I ben più breve del II, VIII allungato, da una volta e mezzo a più di due volte $\mathrm{e}$ mezza più lungo che largo, XI estremamente allungato, con i lati sinuati, ristretti al terzo prossimale, lungo da quasi a più lungo della somma dei tre precedenti (Figg. 13-15).

Pronoto con i lati regolarmente arrotondati, ma anche con accenno di lieve sinuosità appena avanti gli angoli posteriori, che sono acuti, e con la massima larghezza alla base.

Carena mesosternale alta, arrotondata e particolarmente prominente in avanti e con il profilo ventrale da diritto a lievemente curvo e sinuato; apofisi posteriore abbondantemente oltrepassante l'intero metasterno.

Elitre prive di stria suturale, generalmente larghe, 
con la massima larghezza poco distante dalla base e con striole trasversali fitte e ben marcate.

Apparato metatergale piccolissimo e molto breve.

Apice delle tibie munito di speroni interni polidentati, meso e metatibie con cestelli apicali spinosi completi.

Protarsi maschili moderatamente dilatati, di poco più stretti o al massimo larghi quanto l'apice della protibia.

Lobo mediano dell'edeago (Figg. 42 e 43) relativamente piccolo, lungo da un quarto a un terzo del corpo, tozzo; in visione dorsale con i lati paralleli o lievemente convergenti nella metà distale, l'apice di forma ogivale e munito di una linguetta apicale pronunciata e asimmetrica, troncata obliquamente; in visione laterale si presenta regolarmente curvo, robusto, con l'apice assottigliato e piegato verso l'alto a becco d'oca.

Sacco interno con pezzo basale ad $\mathrm{Y}$ atrofico, poco sclerificato e poco evidente; mancano anche fanere mediane sclerificate sostituite da fasci membranosi a volte spinulosi che si prolungano fino all'apice.

Parameri esili, poco più brevi del lobo mediano, in visione dorsale sinuati al terzo basale, muniti di tre corte setole, due propriamente apicali e una appena più arretrata.

Spiculum dell'ottavo ventrite nella femmina quasi inesistente, al massimo ridotto ad un ispessimento calloso mediano appena accennato (Figg. 66 e 67).

Spermateca piccola con la parte prossimale reniforme particolarmente curva e la parte distale sferica e poco voluminosa.

Vi appartengono, allo stato attuale delle conoscenze, le seguenti specie:

Cacciamalia allegrettii (Jeannel, 1930)

Cacciamalia antonellae (Vailati, 1988)

Cacciamalia viallii (Pavan, 1938)

Cacciamalia glacialis glacialis (Vailati, 1975)

Cacciamalia glacialis filicornis (Vailati, 1979)

Cacciamalia glacialis monguzzii (Vailati, 1988)

Cacciamalia glacialis rosai (Vailati, 1988)

DeRIVATIO NOMINIS. Dedico con piacere questo genere alla memoria dell'insigne geologo bresciano Gian Battista Cacciamali (1857-1934), fondatore del Circolo Speleologico "La Maddalena", iniziatore della speleologia esplorativa in terra bresciana sul finire del XIX secolo.
DistribuZione nota. Prealpi Bresciane: M. Guglielmo, media e alta Val Trompia, monti tra la Val Trompia e la Val Sabbia-lago d'Idro; Prealpi Gardesane: media Val Sabbia versante sinistro, gruppo dei M. Pizzocolo-Spino-Marmera, media e alta Val Vestino, Val d'Ampola; Alpi Retiche meridionali: Valle del Caffaro e Giudicaria Inferiore.

genere Viallia Pavan, 1950

(Figg. 16-18, 44, 45, 68)

Viallia Pavan, 1950, Speleon, I (1): 56.

Viallia Pavan: Vailati, 1988: 133.

Viallia Pavan: Newton, 1998: 121.

Viallia Pavan: Perreau, 2000: 254.

Viallia Pavan: Perreau, 2004: 177.

SPECIE TIPO: Viallia Alfanoi Pavan, 1950.

RiDESCRIZIONE. Corpo da batiscioide di media taglia, ellittico o ovoidale, a foleuonoide, coperto da fitta pubescenza relativamente lunga.

Antenne del $\hat{\partial}$, distese all'indietro, raggiungenti dal terzo basale al quinto apicale delle elitre; articolo I ben più breve del II, VIII da poco più lungo a due volte e mezza più lungo che largo, XI lanceolato, allargato nel mezzo, di forma romboidale, e poco più lungo del precedente (Figg. 16-18).

Pronoto, nell'unica specie foleuonoide ( $V$. alfanoi) ben più stretto delle elitre, nelle altre specie largo alla base, con i lati regolarmente arrotondati, ma anche lievemente sinuati appena avanti gli angoli posteriori

Carena mesosternale più bassa e anteriormente sfuggente nella forma foleuonoide, alta, ampiamente arrotondata e molto prominente in avanti nelle specie a facies batiscioide; in queste il lato ventrale, leggermente arcuato, risulta in visione ventrale appiattito, largo, in forma di lunga losanga; apofisi posteriore oltrepassante l'intero metasterno.

Elitre prive di stria suturale e con striole trasversali da poco a ben marcate, con pubescenza particolarmente lunga.

Apparato metatergale piccolo e breve, con la doccia longitudinale da larga quanto lunga a larga come un quarto della lunghezza.

Apice delle tibie munito di speroni interni polidentati, meso e metatibie con cestelli apicali spinosi completi. 
Protarsi maschili brevi e poco dilatati, più dilatati nelle forme batiscioidi, ma sempre più stretti dell'apice della protibia.

Lobo mediano dell'edeago (Figg. 44 e 45) relativamente piccolo lungo meno di un terzo del corpo, tozzo; in visione dorsale con la lama basale ampia e $\mathrm{i}$ lati convergenti verso l'apice regolarmente ogivato ma non appuntito; in visione laterale si presenta con la lama basale lunga, moderatamente curvo e con la metà distale appiattita e dorsalmente sinuosa, con l'apice piegato ventralmente.

Sacco interno con pezzo basale ad $\mathrm{Y}$ atrofico, poco sclerificato e poco evidente, senza alcuna fanera mediana sclerificata, ma fornito al centro di fasci membranosi e setoso-spinulosi.

Parameri di normale robustezza, più brevi del lobo mediano e muniti di due setole apicali di uguale lunghezza e di una preapicale più arretrata.

Spiculum dell'ottavo ventrite nella femmina poco o nulla sviluppato, il margine anteriore del ventrite presenta un lieve risalto ampiamente ottuso (Fig. 68).

Spermateca fortemente asimmetrica, con la parte prossimale voluminosa e la distale molto piccola, entrambe con le pareti sottili e poco sclerificate.

Vi appartengono, allo stato attuale delle conoscenze, le seguenti specie:

Gruppo «alfanoi» Vailati, 1988

Viallia alfanoi Pavan, 1950

Gruppo «mismae» Vailati, 1988

Viallia mismae (Inzaghi \& Regalin, 1982)

Viallia grottoloi Vailati, 1988

Viallia cappai Vailati, 1988

DistribuZIONE NOTA. Prealpi Bergamasche: Valle Imagna, media Valle Brembana, bassa e media Valle Seriana, M. Pora.

genere Monguzziella Vailati, 1993

(Figg. 19, 46, 69, 86)

Monguzziella Vailati, 1993, Natura Bresciana, 28: 262.

Monguzziella Vailati: Newton, 1998: 117.

Monguzziella Vailati: Perreau, 2000: 253.

Monguzziella Vailati: Perreau, 2004: 166.

SPECIE TIPO: Monguzziella grottoloi Vailati, 1993.
RIDESCRIZIONE. Corpo batiscioide di piccola taglia, ma allungato e con avancorpo ristretto, coperto da fitta pubescenza relativamente lunga.

Antenne del $\widehat{\partial}$ relativamente brevi, distese all'indietro, raggiungenti quasi la metà delle elitre; articolo I più breve del II, VIII trasverso, XI piriforme, lungo come la somma del precedente più metà del IX (Fig. 19).

Pronoto trasverso, ma più stretto della base delle elitre, con i lati regolarmente arrotondati, non sinuosi ma ristretti alla base, con la massima larghezza al terzo basale.

Carena mesosternale particolarmente bassa, con profilo anteriore non prominente, sfuggente, ventralmente arrotondato e senza dente, con profilo grossolanamente seghettato; apofisi posteriore prolungata sopra il metasterno, di cui non raggiunge il bordo posteriore.

Elitre prive di stria suturale e con striole trasversali ben evidenti ma superficiali.

Apparato metatergale piccolo e breve, con la doccia longitudinale larga la metà della lunghezza.

Apice delle tibie munito di speroni interni polidentati, meso e metatibie con cestelli apicali spinosi completi.

Protarsi maschili moderatamente dilatati, larghi poco più dei due terzi dell'apice della protibia.

Lobo mediano dell'edeago (Fig. 46) lungo circa un terzo del corpo, tozzo e robusto; in visione dorsale con i lati paralleli e con una evidente strozzatura preapicale, con l'apice arrotondato, lateralmente dilatato e spianato, munito di una linguetta apicale breve e arrotondata; in visione laterale si presenta robusto, regolarmente curvo, con l'apice diritto.

Sacco interno con pezzo basale ad Y piccolo, semplice, debolmente sclerificato; regione mediana priva di particolari fanere sclerificate, ma solo con strutture membranose coperte da piccolissime spinule; distalmente si dipartono due liste sinuose e divergenti, debolmente sclerificate.

Parameri lunghi, raggiungenti l'apice del lobo mediano, assottigliati al quarto distale, muniti di due piccolissime setole apicali e una ventrale più arretrata.

Ottavo ventrite nella femmina con il bordo anteriore privo di spiculum (Fig. 69).

Spermateca asimmetrica, con la parte prossimale più voluminosa e allungata e la parte distale sferica $\mathrm{e}$ più piccola.

Vi appartiene, allo stato attuale delle conoscenze, solo: 
Monguzziella grottoloi Vailati, 1993

DistribuZIONE NOTA. Prealpi Venete occidentali: Altopiani di Tonezza-Folgaria e Vigolana, Altopiano di Lavarone-Luserna, Gruppo del Carega-Piccole Dolomiti-Pasubio e Monti Lessini veronesi (Piva, 2016).

genere Hartigiella Müller, 1935 genere valido (Figg. 20, 47, 70)

Bathysciola (Hartigia) Müller, 1928, Studi trent. Sci. nat., IX: 188.

Bathysciola (Hartigiella) nom. nov.: Müller, 1935: 62. Boldoria «gruppo baldensis»: Vailati, 1988: 188.

Boldoria Jeannel (pars): Newton, 1998: 114.

Boldoria groupe «baldensis »: Perreau, 2000: 250.

Boldoria Jeannel (pars): Perreau, 2004: 162.

SPECIE TIPO: Bathysciola (Hartigia) baldensis Müller, 1928.

RIDESCRIZIONE. Corpo batiscioide di media taglia, ovale, attenuato posteriormente.

Antenne del ô esili, lunghe, raggiungenti, distese all'indietro, la metà delle elitre; articolo I ben più breve del II, VIII più di due volte più lungo che largo, XI lanceolato e simile, anche se meno allungato, a quello di Cacciamalia, cioè con i lati sinuati, ristretti al terzo prossimale, lungo come il precedente più metà del IX (Fig. 20).

Pronoto con i lati regolarmente arrotondati, con la massima larghezza alla base o appena avanti a questa.

Carena mesosternale alta, ampiamente arrotondata e prominente in avanti e con il profilo ventrale leggermente curvo; apofisi posteriore lunga, oltrepassante l'intero metasterno.

Elitre ovoidali, prive di stria suturale e con striole trasversali regolari e ben marcate.

Apparato metatergale alquanto ridotto, la doccia longitudinale è larga quanto lunga.

Apice delle tibie munito di speroni interni polidentati, meso e metatibie con cestelli apicali spinosi completi.

Protarsi maschili particolarmente sviluppati in lunghezza, lunghi quanto e anche più lunghi della protibia, molto dilatati, con il primo tarsomero ben più largo della protibia.
Lobo mediano dell'edeago (Fig. 47) lungo poco più di un terzo del corpo, tozzo; in visione dorsale con i lati subparalleli nella metà basale e quindi convergenti verso l'apice, che è regolarmente ogivato e terminato da una piccolissima linguetta apicale arrotondata; in visione laterale si presenta ottusamente piegato, con l'apice assottigliato e curvato verso il basso.

Sacco interno con pezzo basale ad Y ben sviluppato e sclerificato; fanera mediana costituita da una placchetta centrale sclerificata "a farfalla" circondata da strutture ialine fibrose e distalmente con due liste sclerificate subparallele fra le quali vi sono alcune lunghe spine e due strutture a superficie cosparsa da piccoli denti.

Parameri lunghi ed estremamente esili, poco più brevi del lobo mediano, muniti di tre setole, una propriamente apicale, e due preapicali, delle quali quella più prossimale piccolissima.

Spiculum dell'ottavo ventrite nella femmina piccolo, tozzo poco sviluppato in lunghezza e con l'apice appuntito (Fig. 70).

Spermateca con la parte prossimale voluminosa, piriforme, allungata e la parte distale sferica e più piccola.

Vi appartiene, allo stato attuale delle conoscenze, solo:

Hartigiella baldensis (Müller, 1928)

DistribuZIONE NOTA. Prealpi Gardesane: tutto il massiccio del M. Baldo, M. Stivo e fino al Bondone.

genere Ghidinia Pavan, 1939 genere valido

(Figg. 21, 48, 49, 71, 72, 87, 88)

Ghidinia Pavan, 1939, Mem. Soc. entom. it., 18: 106. Boldoria «gruppo morettii»: Vailati, 1988: 194.

Boldoria Jeannel (pars): Newton, 1998: 114.

Boldoria groupe «morettii»: Perreau, 2000: 253.

Boldoria Jeannel (pars): Perreau, 2004: 162.

SPECIE TIPO: Ghidinia Morettii Pavan, 1939.

RidesCRIZIONE. Corpo batiscioide di taglia mediogrande, ellittico e alquanto allungato, fortemente attenuato in addietro.

Antenne del $\widehat{\partial}$ lunghe ed esili, distese all'indietro, quasi raggiungenti l'apice delle elitre; rapporto di lun- 
ghezza dei primi due articoli variabile, ma tendenzialmente sono di uguale lunghezza, l'articolo I può essere appena più breve, uguale $\mathrm{o}$ appena più lungo del II, VIII allungato, da poco meno a più di quattro volte più lungo che largo, XI lanceolato e con i lati sinuati al terzo basale, lungo come la somma del precedente più metà del IX (Fig. 21).

Pronoto campaniforme, con i lati leggermente sinuati prima degli angoli posteriori, che sono acuti, e con la massima larghezza alla base.

Carena mesosternale alta, ampiamente arrotondata e alquanto prominente in avanti, con il profilo ventrale da diritto a leggermente sinuato; apofisi posteriore oltrepassante l'intero metasterno.

Elitre particolarmente attenuate posteriormente, con la massima larghezza al quarto o al terzo basale, prive di stria suturale e con striole trasversali fitte e numerose, superficiali ma ben evidenti.

Apparato metatergale piccolo e breve, con la doccia longitudinale larga da un quinto a metà della lunghezza.

Apice delle tibie munito di speroni interni polidentati, meso e metatibie con cestelli apicali spinosi completi.

Protarsi maschili ben dilatati, larghi circa come l'apice della protibia.

Lobo mediano dell'edeago (Figg. 48 e 49) relativamente grande, lungo quasi un terzo del corpo; in visione dorsale con i lati paralleli o lievemente sinuati e l'apice subtriangolare, tozzo; in visione laterale si presenta ampiamente curvati e con l'apice assottigliato, da diritto a leggermente curvato dorsalmente.

Sacco interno con pezzo basale ad $\mathrm{Y}$ ben sclerificato; fanere mediane costituite da una placchetta centrale sclerificata e da una struttura con braccia rivolte prossimalmente, il tutto circondato da fasci setoso-spinulosi; distalmente sono presenti due liste sclerificate subparallele, sinuose.

Parameri esili, ben più brevi del lobo mediano, muniti all'apice di tre setole di diversa lunghezza, di cui una molto piccola.

Spiculum dell'ottavo ventrite nella femmina piccolo, breve ma ben conformato all'apice, che può essere arrotondato ma anche bilobato (Figg. 71 e 72).

Spermateca con la parte prossimale reniforme molto allungata e curva e la parte distale sferica e voluminosa; ductus distalmente non sclerificato.

Vi appartengono, allo stato attuale delle conoscenze, le seguenti specie:
Ghidinia aguinensis Vailati, 1974

Ghidinia morettii morettii Pavan, 1939

Ghidinia morettii vesallae Vailati, 1974

Ghidinia vailatii Cavadini, 1988

DistribuZione nota. Prealpi Bergamasche: Sebino bergamasco; Prealpi Bresciane occidentali: a nord massiccio del M. Guglielmo, a sud zona di Punta dell'Orto e dintorni di Vesalla.

genere Insubriella Vailati, 1990

(Figg. 22, 50, 73)

Insubriella Vailati, 1990, Natura Bresciana, 25: 214.

Insubriella Vailati: Newton, 1998: 116.

Insubriella Vailati: Perreau, 2000: 253.

Insubriella Vailati: Perreau, 2004: 166.

SPECIE TIPO: Insubriella paradoxa Vailati, 1990.

RIDESCRIZIONE. Corpo tendenzialmente foleuonoide, allungato, attenuato posteriormente.

Antenne del $\widehat{\partial}$ lunghe, sottili, distese all'indietro raggiungenti il quarto apicale delle elitre; articolo I ben più lungo del II, VIII cilindrico, allungato, più di quattro volte più lungo che largo, XI lanceolato, leggermente ristretto al terzo basale e lungo come la somma del precedente più un quarto del IX (Fig. 22).

Pronoto trasverso, ma stretto, con i lati sinuosi, più o meno bruscamente ristretti al terzo basale, ugualmente largo a metà quanto alla base nel maschio, più largo alla base nella femmina e con gli angoli posteriori acuti.

Carena mesosternale bassa e affilata, arrotondata ma non prominente in avanti, sfuggente e con il profilo ventrale sprovvisto di dente, ma leggermente incavato; apofisi posteriore prolungata sopra tutto il metasterno.

Elitre prive di stria suturale e con striole trasversali fitte, regolari e ben marcate.

Apparato metatergale piccolissimo, semplice, con la doccia longitudinale larga un terzo della lunghezza.

Apice delle tibie munito di speroni interni polidentati, meso e metatibie con cestelli apicali spinosi completi.

Protarsi maschili lunghi e ben dilatati, larghi quanto l'apice della protibia.

Lobo mediano dell'edeago (Fig. 50) lungo meno 
di un terzo del corpo, tozzo, robusto; in visione dorsale con i lati paralleli e l'apice di forma ogivale e appuntito, munito di linguetta apicale pronunciata; in visione laterale si presenta regolarmente curvo, molto robusto nella metà basale, più assottigliato verso l'apice, che è curvato verso l'alto a becco d'oca.

Sacco interno con pezzo basale ad Y ben sviluppato e sclerificato; fanere mediane costituite da una placchetta centrale sclerificata con due braccia rivolte prossimalmente e distalmente, con due liste distali sclerificate subparallele munite alla base di fascetti spinulosi.

Parameri di normale robustezza, non raggiungenti l'apice del lobo mediano, muniti di due setole apicali di eguale lunghezza, ma di cui una particolarmente robusta e sclerificata, e una appena più arretrata, più breve.

Spiculum dell'ottavo ventrite nella femmina molto piccolo, con l'apice tronco, sub quadrato (Fig. 73).

Spermateca grande, asimmetrica con la parte prossimale sviluppata in lunghezza e la parte distale sferica e più voluminosa.

Vi appartiene, allo stato attuale delle conoscenze, solo:

Insubriella paradoxa Vailati, 1990

DistribuZIONE NOTA. Prealpi Bresciane: nota di poche stazioni sui rilievi del territorio di Bione e di Casto.

genere Ragazzonia $\mathrm{n}$. gen.

(Figg. 23, 24, 51, 52, 74, 75, 89, 90)

Boldoria «gruppo breviclavata» Vailati, 1988: 212.

Boldoria Jeannel (pars): Newton, 1998: 114.

Boldoria groupe «breviclavata»: Perreau, 2000: 250.

Boldoria Jeannel (pars): Perreau, 2004: 162.

SPECIE TIPO: Bathysciola (Boldoria) breviclavata Müller, 1931, Atti Mus. civ. St. nat. Trieste, 11 (2): 197.

Descrizione. Corpo batiscioide di medie dimensioni, ellittico e tozzo, globoso.

Antenne del $\hat{\partial}$ brevi, fortemente clavate, distese all'indietro raggiungenti il quinto o al massimo il quarto basale delle elitre; articolo I tozzo, ben più breve del II, VIII da trasverso ad appena più lungo che largo, XI piriforme, largo, sempre più breve della somma dei due precedenti (Figg. 23 e 24).

Pronoto largo, convesso, con i lati regolarmente arrotondati, con la massima larghezza alla base.

Carena mesosternale moderatamente alta, poco prominente in avanti e con il profilo ventrale diritto o poco sinuoso; apofisi posteriore libera, oltrepassante l'intero metasterno.

Elitre globose, ampiamente arrotondate e con massima larghezza al quarto basale; prive di stria suturale e con striole trasversali distanziate, grossolane, ma ben marcate.

Apparato metatergale piccolo e breve, con la doccia longitudinale poco sviluppata in lunghezza.

Apice delle tibie munito di speroni interni polidentati, meso e metatibie con cestelli apicali spinosi completi.

Protarsi maschili ben dilatati, larghi come o più larghi dell'apice della protibia.

Lobo mediano dell'edeago (Figg. 51 e 52) grande, lungo circa la metà del corpo, tozzo; in visione dorsale con i lati leggermente sinuosi e l'apice arrotondato e terminante con una piccolissima linguetta; in visione laterale si presenta ottusamente piegato, robusto, con l'apice da diritto a bruscamente flesso in basso.

Sacco interno con pezzo basale ad Y grande e ben sclerificato; fanera mediana grande, complessa, con braccia sclerificate rivolte prossimalmente e circondata da strutture spinulose; distalmente con due liste sclerificate simmetriche e divergenti verso l'apice.

Parameri esili, ben più brevi del lobo mediano, muniti all'apice di tre setole di diversa lunghezza.

Spiculum dell'ottavo ventrite nella femmina piccolo, tozzo, ridotto in lunghezza e con l'apice da arrotondato a largo e quadrato (Figg. 74 and 75).

Spermateca con la parte prossimale allungata, la parte mediana alquanto breve e la distale da subsferica ad allungata, ma sempre meno voluminosa; ductus distalmente sclerificato per breve tratto.

Vi appartengono, allo stato attuale delle conoscenze, le seguenti specie:

Ragazzonia vestae vestae (Ghidini, 1936)

Ragazzonia vestae meridionalis (Vailati, 1988)

Ragazzonia trumplina (Vailati, 1988)

Ragazzonia breviclavata (Müller, 1931)

DeRIVATIO NOMINIS. Questo genere è dedicato alla me- 
moria del geologo e paleontologo bresciano Giuseppe Ragazzoni (1824-1898), maestro dello stesso Gian Battista Cacciamali e compagno di escursioni dell'Abate Antonio Stoppani, che per primo ebbe a visitare, per indagini paleontologiche, numerose grotte del Bresciano.

DistribuZione nota. Prealpi Bresciane: M. Guglielmo, media Val Trompia, monti tra la Val Trompia e la Val Sabbia fino al pedemonte, dintorni del lago d'Idro; Prealpi Gardesane: media Val Sabbia e lago d'Idro versante sinistro, gruppo dei M. PizzocoloSpino-Marmera, media e alta Val Vestino, Val d'Ampola; Alpi Retiche meridionali: Valle del Caffaro e Giudicaria Inferiore. Nella parte settentrionale ricalca l'areale occupato dal genere Cacciamalia.

genere Miettiella Piva, 2016

(Figg. 25, 53, 76)

SPECIE TIPO: Miettiella vespertilio Piva, 2016, Boll. Soc. entom. It., 148: 63.

RIDESCRIZIONE. Corpo batiscioide, di piccola taglia, ellittico e convesso.

Antenne del $\widehat{\partial}$ relativamente allungate, clavate, distese all'indietro quasi raggiungenti il terzo basale delle elitre; articolo I più breve del II, VIII una volta e mezzo più lungo che largo, XI piriforme, largo, sempre più breve della somma dei due precedenti (Fig. 25).

Pronoto largo, convesso, con i lati regolarmente arrotondati, con la massima larghezza presso la base.

Carena mesosternale moderatamente alta, non prominente in avanti e con il profilo anteriore poco arrotondato, obliquamente sfuggente, e quello ventrale diritto o poco sinuoso; apofisi posteriore libera, prolungata solo per breve tratto sul metasterno.

Elitre brevi e ampie, ampiamente arrotondate e con massima larghezza circa a metà; prive di stria suturale e con striole trasversali ben evidenti.

Apparato metatergale piccolo e breve, con la doccia longitudinale poco sviluppata in lunghezza.

Apice delle tibie munito di speroni interni polidentati, meso e metatibie con cestelli apicali spinosi completi.

Protarsi maschili ben dilatati, di poco più larghi dell'apice della protibia.

Lobo mediano dell'edeago (Fig. 53) piccolo, lungo circa un terzo del corpo, tozzo; in visione dorsale con i lati leggermente sinuosi e l'apice largo, arrotondato e terminante con una piccolissima linguetta arrotondata; in visione laterale si presenta poco curvo, robusto, con l'apice lievemente flesso in basso.

Sacco interno con pezzo basale ad Y piccolo e poco definito; fanera mediana complessa, grande e ben sclerificata, riproducente la sagoma di un chirottero.

Parameri esili, più brevi del lobo mediano, muniti all'apice di tre setole di diversa lunghezza.

Spiculum dell'ottavo ventrite nella femmina piccolo, poco sviluppato in lunghezza e con l'apice arrotondato (Fig. 76).

Spermateca con la parte prossimale allungata, la parte mediana brevissima, quasi inesistente, e la distale piccola e sferica.

Vi appartiene, allo stato attuale delle conoscenze, solo:

Miettiella vespertilio Piva, 2016

DistribuZione nota. Prealpi Venete occidentali: attualmente nota di una sola stazione negli Alti Lessini veronesi (Piva, 2016).

genere Boldoria Jeannel, 1924

(Figg. 26, 54, 77, 91)

Bathysciola (Boldoria) Jeannel, 1924, Arch. Zool. expér. gén., 63: 117.

Boldoria «gruppo aculeata» Vailati, 1988: 231.

Boldoria Jeannel (pars): Newton, 1998: 114.

Boldoria groupe «aculeata»: Perreau, 2000: 249.

Boldoria Jeannel (pars): Perreau, 2004: 162.

SPECIE TIPO: Bathysciola (Boldoria) aculeata Jeannel, 1924, Arch. Zool. expér. gén., 63: 117.

RIDESCRIZIONE. Corpo batiscioide, di piccola taglia, ellittico allungato.

Antenne del $\widehat{\partial}$, distese all'indietro, raggiungenti il terzo basale delle elitre; articolo I ben più breve del II, VIII di poco più lungo che largo, XI lanceolato e lungo come la somma dei due precedenti più metà dell'VIII (Fig. 26).

Pronoto con i lati regolarmente arrotondati, con la massima larghezza avanti la base che è leggermente ristretta.

Carena mesosternale alta, arrotondata ma poco 
prominente in avanti e con il profilo ventrale diritto; apofisi posteriore oltrepassante l'intero metasterno.

Elitre prive di stria suturale e con striole trasversali fini ma ben marcate.

Apparato metatergale piccolo e brevissimo, con la doccia longitudinale larga da metà a un terzo della lunghezza.

Apice delle tibie munito di speroni interni polidentati, meso e metatibie con cestelli apicali spinosi completi.

Protarsi maschili brevi e poco dilatati, più stretti dell'apice della protibia.

Lobo mediano dell'edeago (Fig. 54) lungo un terzo del corpo, tozzo; in visione dorsale con i lati paralleli e l'apice di forma ogivale e appuntito; in visione laterale si presenta ottusamente piegato, robusto, con l'apice non troppo assottigliato e quasi diritto, di pochissimo flesso in basso.

Sacco interno con pezzo basale ad $\mathrm{Y}$ ben sclerificato e complesso; fanere mediane costituite da una placchetta centrale sclerificata sormontata da una struttura bilobata con due braccia rivolte prossimalmente e distalmente con due fasci simmetrici e paralleli di spinule; assenti strutture apicali sclerificate.

Parameri robusti, poco più brevi del lobo mediano, muniti di tre setole circa equivalenti in lunghezza, due propriamente apicali e una appena più arretrata.

Spiculum dell'ottavo ventrite nella femmina piccolo, ridotto ad una linguetta triangolare poco sviluppata in lunghezza e con l'apice arrotondato (Fig. 77).

Spermateca con la parte prossimale poco allungata e la parte distale sferica e più voluminosa; ductus distalmente sclerificato per breve tratto.

Vi appartiene, allo stato attuale delle conoscenze, solo:

Boldoria aculeata (Jeannel, 1924)

DistriBuZIONE NOTA. Prealpi Bresciane: nota di numerose stazioni concentrate in un areale alquanto limitato, nel gruppo dei monti Palosso e Doppo.

genere Pavaniola n. gen.

(Figg. 27-29, 55-57, 78, 79, 92, 93)

Boldoria «gruppo ghidinii» Vailati, 1988: 236.

Boldoria Jeannel (pars): Newton, 1998: 114.
Boldoria groupe «ghidinii»: Perreau, 2000: 251.

Boldoria Jeannel (pars): Perreau, 2004: 162.

SpeCIE TIPO: Bathysciola (Boldoria) Ghidinii Lona, 1937, Boll. Soc. entom. it., 69 (5-6): 92.

Descrizione. Corpo batiscioide, di piccola taglia, ellittico allungato.

Antenne del $\widehat{\partial}$, distese all'indietro, raggiungenti il terzo basale delle elitre; articolo I sempre ben più breve del II, VIII da trasverso ad appena più lungo che largo, XI lanceolato e lungo come o poco meno la somma dei due precedenti (Figg. 27-29).

Pronoto con i lati regolarmente arrotondati, con la massima larghezza presso la base.

Carena mesosternale alta, arrotondata ma poco o nulla prominente in avanti e con il profilo ventrale diritto; apofisi posteriore oltrepassante l'intero metasterno.

Elitre prive di stria suturale e con striole trasversali regolari e ben marcate.

Apparato metatergale piccolo e particolarmente breve, con la doccia longitudinale da tanto larga quanto lunga a larga un quinto della lunghezza.

Apice delle tibie munito di speroni interni polidentati, meso e metatibie con cestelli apicali spinosi completi.

Protarsi maschili brevi, da poco a moderatamente dilatati, sempre ben più stretti dell'apice della protibia.

Lobo mediano dell'edeago (Figg. 55-57) piccolo, lungo da un quarto a poco meno di un terzo del corpo, tozzo; in visione dorsale con i lati paralleli e l'apice di forma ogivale e appuntito, ma non acuminato; in visione laterale si presenta ottusamente piegato, robusto, con la metà distale leggermente sinuosa e con l'apice non troppo assottigliato e leggermente flesso in basso.

Sacco interno con pezzo basale ad $\mathrm{Y}$ ben sclerificato e semplice; fanere mediane costituite da una placchetta centrale sclerificata, circondata da strutture setoso-spinulose e dentate e distalmente con strutture membranose setose; sono assenti strutture apicali sclerificate.

Parameri robusti, ben più brevi del lobo mediano, di norma muniti all'apice di quattro setole (tre nel caso di $P$. regalini) sempre particolarmente lunghe e flagelliformi.

Spiculum dell'ottavo ventrite nella femmina piccolo, ridotto ad una tozza linguetta poco sviluppata in lunghezza e con l'apice allargato e bilobato (Fig. 78 e 79).

Spermateca piccola, con la parte prossimale reni- 
forme piccola, la parte distale sferica e più voluminosa e la parte mediana molto breve.

Vi appartengono, allo stato attuale delle conoscenze, le seguenti specie:

Gruppo «ghidinii» Vailati, 1988

Pavaniola longitarsis (Pavan, 1941)

Pavaniola ghidinii ghidinii (Lona, 1937)

Pavaniola ghidinii polavenensis (Pavan, 1939)

Pavaniola ghidinii blesioi (Vailati, 1988)

Pavaniola ghidinii kahleni (Vailati, 1988)

Gruppo «comottii» nov. (descrizione nella Chiave dei generi)

Pavaniola comottii (Vailati, 1988)

Gruppo «regalini» nov. (descrizione nella Chiave dei generi)

Pavaniola regalini (Vailati, 1988)

DeRIVATIO NOMINIS. Dedico con grande piacere questo genere alla memoria del compianto Prof. Mario Pavan, la cui intensa attività speleologica e biospeleologica, svolta durante i suoi anni giovanili in compagnia degli indimenticabili Corrado Allegretti, Leonida Boldori e Gian Maria Ghidini, fu di fondamentale importanza quale solida base per gli sviluppi che avrebbe avuto la ricerca biospeleologica nelle Prealpi lombarde.

DistribuZIONE NOTA. Prealpi Bergamasche: tra la bassa Val Seriana e la Val Cavallina e nel Sebino bergamasco. Prealpi Bresciane: monti tra il lago d'Iseo e la media e bassa Val Trompia, pedemonte presso la città di Brescia, gruppo del M. Palosso e media Val Sabbia.

\section{CONSIDERAZIONI BIOEOGRAFICHE}

Le cartine riportate nelle Figg. 94 e 95 mostrano gli areali dei generi, già definiti in margine ad ogni genere trattato alla voce "distribuzione nota". Come appare evidente, alcuni generi della «serie filetica di Dellabeffaella», in particolare i generi Dellabeffaella, Canavesiella e Archeoboldoria sono, secondo le attuali conoscenze, ben delimitati e fra loro disgiunti, distribuiti lungo il margine prossimo al pedemonte del settore alpino più occidentale, mentre il genere Stop-

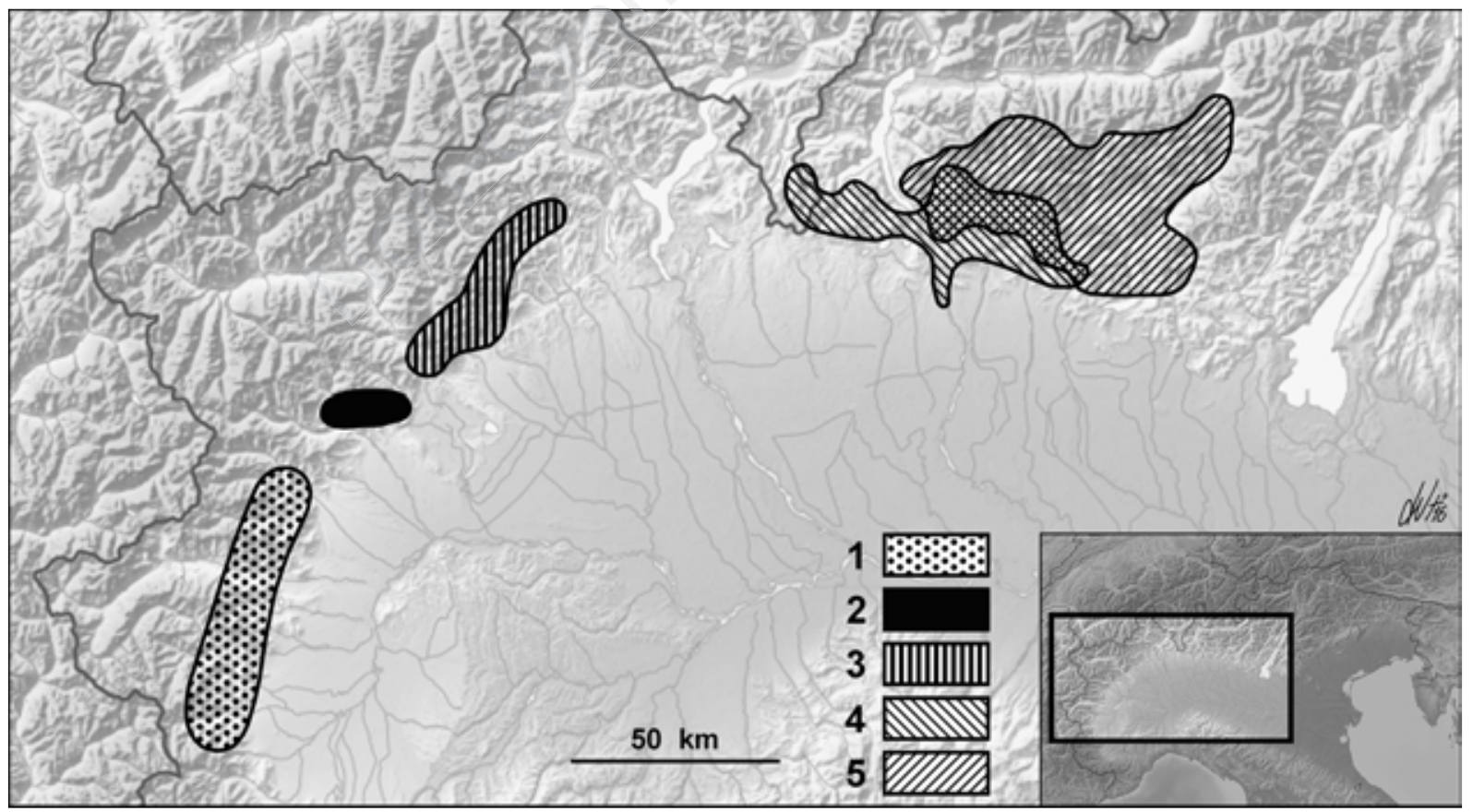

Fig. 94. Areali dei generi della "serie filetica di Dellabeffaella". 1: Dellabeffaella; 2: Canavesiella; 3: Archeoboldoria; 4: Stoppaniola n. gen.; 5: Pseudoboldoria. 
paniola, distribuito nelle Prealpi Comasche e Bergamasche, risulta in parte sovrapposto al genere Pseudoboldoria, ampiamente presente nelle Alpi Orobie e Prealpi Bergamasche (marginalmente verso Est anche nelle Prealpi Bresciane). Questi ultimi due generi poi, si sovrappongono ad alcuni altri della «serie filetica di Boldoria», in particolare al genere Viallia nelle Prealpi Bergamasche, e al genere Ghidinia, distribuito tra queste e le Prealpi Bresciane nell'area sebina, delimitato tra la Val Cavallina e la Valle Trompia. Oltre a quest'ultimo genere, nelle Prealpi Bresciane e Gardesane si assiste alla maggiore complessità distributiva (vedi cartine), con i generi Pavaniola, Cacciamalia, Insubriella, Boldoria e Ragazzonia, che mostrano areali variamente sovrapposti, almeno in parte, mentre Hartigiella, che occupa l'intero massiccio del Baldo e risale verso Nord fino al Bondone, risulterebbe delimitato a Est dalla valle dell'Adige, ben disgiunto dai generi Monguzziella e Miettiella, noti finora dei Lessini Veronesi e del basso Trentino.

I complessi che in passato erano stati descritti come "gruppi di specie" e attribuiti a due soli generi
- Boldoria e Pseudoboldoria - riflettevano una pretesa maggiore omogeneità di tali taxa che, allo stato attuale, non risulta più essere soddisfatta da una visione più analitica. La scelta di istituire i nuovi generi sopra trattati è giustificata non solo dall'analisi dei caratteri diacritici messi in evidenza, ma anche dalla convinzione che il nuovo assetto tassonomico appaia con valore informativo maggiore circa la biodiversità del popolamento a Leptodirinae dell'area interessata. Un popolamento che appare sempre più complesso $\mathrm{e}$ che, se da un lato si arricchisce di continui nuovi apporti - anche alla luce delle più recenti scoperte (Piva, 2016) - dall'altro non fa che confermare ipotesi già espresse a più riprese anche in altre sedi.

In particolare, la bordura pedemontana delle Prealpi meridionali ha da sempre costituito un formidabile laboratorio di analisi per la comprensione delle vicende paleogeografiche e paleoclimatiche coinvolte nella storia evolutiva e distributiva di questi endemiti e non solo di questi. Basti pensare alla straordinaria ricchezza della fauna a costumi sotterranei più o meno specializzati in molteplici altri gruppi zoologici pre-
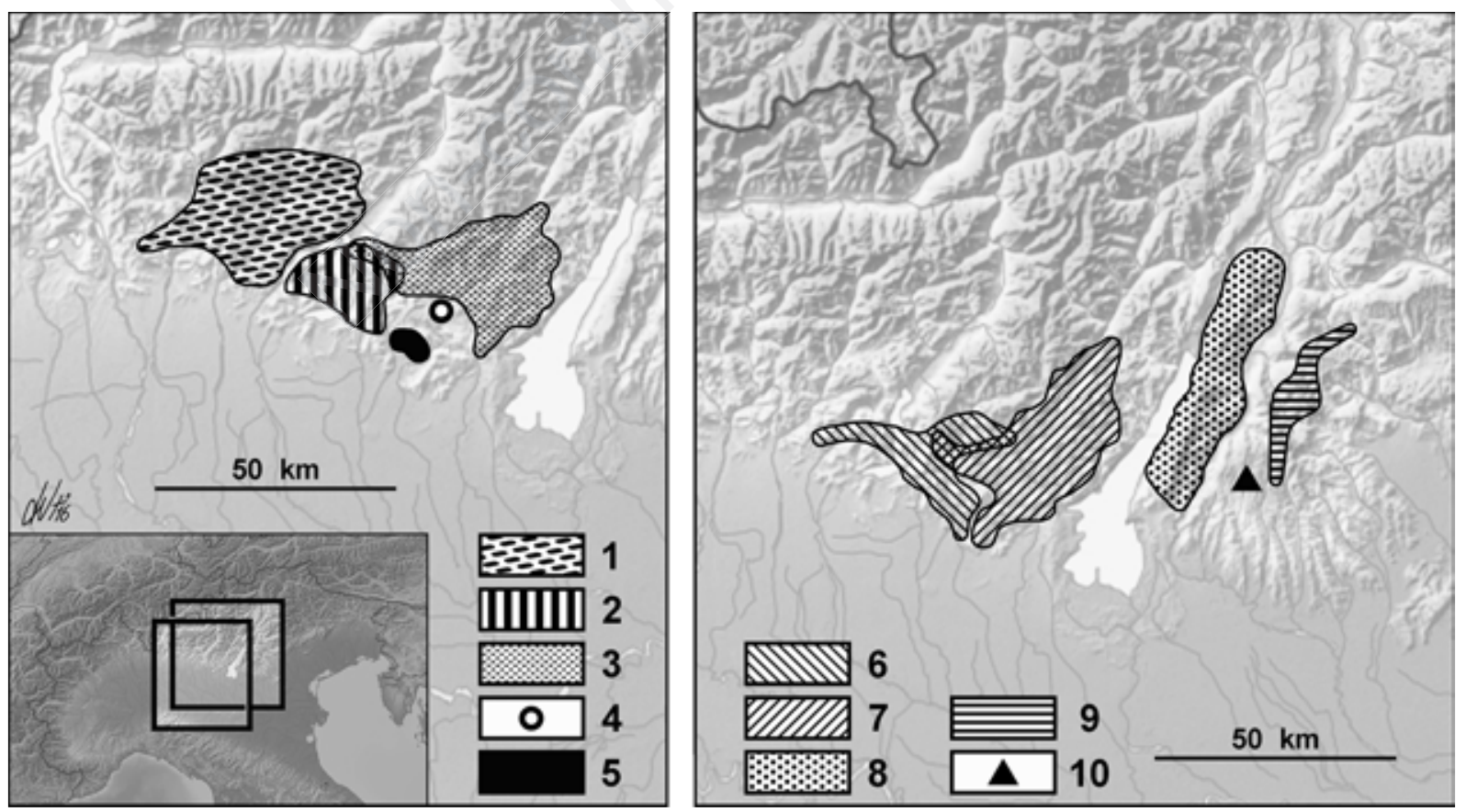

Fig. 95. Areali dei generi della "serie filetica di Boldoria". 1: Viallia; 2: Ghidinia; 3: Cacciamalia; 4: Insubriella; 5: Boldoria; 6: Pavaniola n. gen.; 7: Ragazzonia n. gen.; 8: Hartigiella; 9: Monguzziella; 10: Miettiella. 
senti nell'area: si possono citare, a titolo di esempio, rappresentanti di un'altra famiglia di coleotteri, i Carabidi, con i Trechini dei generi Allegrettia, Boldoriella, Italaphaenops, Orotrechus o Lessinodytes (Casale \& Vigna Taglianti, 2005). Le attuali distribuzioni che ci sono note, mostrano una situazione complessa sulla quale, in passato, ma anche recentemente, si è molto discusso, a torto o a ragione, circa il ruolo di certe presunte barriere o di presunti limiti di areali di raggruppamenti generici e sopragenerici.

Emblematico è l'esempio che ci viene fornito dal massiccio del Monte Baldo, che rappresenta una sorta di "zona grigia" interposta fra la valle del fiume Adige e il lago di Garda, due presunte o "pretese" barriere, alternativamente a più riprese ritenute tali, fino al momento in cui nuove emergenze confermavano o smentivano le interpretazioni di volta in volta sostenute. Ad esempio, fintanto che Hartigiella veniva considerato incluso nel genere Boldoria (Vailati, 1976, 1977), poteva apparire plausibile rimarcare il ruolo di barriera della valle dell'Adige - fatto oltretutto da sempre storicamente sostenuto (Ruffo, 1938, 1950; Vailati, 1976, 1988; Caoduro et al., 1994) - a est della quale sono distribuiti generi di Leptodirini infraflagellati "teleomorfi" (sensu Giachino et al., 1998) delle linee filetiche di Neobathyscia, di Aphaotus e di Aphaobius, (Sbordoni et al., 1982; Zoia, 1998; Vailati, 1993a; Giachino \& Vailati, 2005); la scoperta sul Baldo di Orotrechus vicentinus martinellii Daffner, 1987 e più recentemente di Aphaotus martinellii Giachino \& Vailati, 2005 - due elementi fra i Carabidi Trechini e i Colevidi Leptodirini appartenenti a generi tipicamente distribuiti a est della valle dell'Adige - ci porta però a tenere in considerazione la loro presenza, con le implicazioni del caso, anche a ovest di tale valle, per non parlare di Lessinodytes glacialis Vigna Taglianti \& Sciaky, 1988 (Carabidae Trechinae), scoperto nel Bresciano, sull'altopiano di Cariadeghe, terza specie di un genere prima noto solo dei Lessini (Vigna Taglianti, 1982; Vigna Taglianti \& Sciaky, 1988; Sciaky \& Vigna Taglianti, 1990). Al contrario, ma analogamente, va considerata la presenza a est dell'Adige di generi come Monguzziella e Miettiella, vicini alla più "occidentale" "serie filetica di Boldoria» (Vailati, 1993a; Piva, 2016). D'altra parte, sono ben noti molti altri casi di distribuzioni non solo di Coleotteri, ma anche di numerosi altri Artropodi che, presenti sul Baldo, sono anche diffusi ora a occidente, ora a oriente di tale massiccio, facendo assumere ad esso, in un certo senso, un ruolo di "cerniera" fra due diverse aree zoogeografiche (Ruffo, 1938; Chemini \& Tamanini, 1981; Minelli \& Ruffo, 1989; Vailati, 1993a).

La complessità di queste distribuzioni ci induce, ancora una volta, a riflettere sui possibili eventi che le hanno in qualche modo favorite, se non determinate, che sono da considerare, come già più volte ribadito anche nei lavori sopra citati, certamente eterocroni. È indubbio che spesso lo status di "barriera" non è una condizione permanente, ma variabile nel tempo, in quanto in buona misura dipendente da variabili, ancorché ciclici, episodi paleogeografici e paleoclimatici locali, come le transizioni climatiche glaciali/interglaciali e i conseguenti eventi di possibile mutamento degli ecosistemi forestali. La molteplicità di alterne vicende, temporalmente distinte, ha di certo rimaneggiato precedenti distribuzioni, favorendo in momenti diversi il superamento di possibili barriere precedentemente interposte e fenomeni di vicarianza in un mosaico di accantonamenti eterocroni. In molti casi, si possono osservare situazioni riferibili a episodi alquanto recenti, come l'alternanza dei numerosi cicli glaciali/interglaciali pleistocenici, più volte evocati per spiegare cladogenesi per allopatria di taxa di livello specifico o subspecifico, come ad esempio illustrato nei modelli distributivi delle specie del genere Aphaotus in Giachino \& Vailati (2005) o, ancora, in Vailati (1988) per le specie e sottospecie delle "Boldoria del gruppo glacialis" (ora Cacciamalia n. gen.), modelli esemplificativi, ma facilmente applicabili anche alle specie di altri generi. I rilievi della bordura prealpina prossimi al pedemonte padano, risparmiati dalla copertura glaciale della calotta alpina, ma allo stesso tempo frammentati dalle numerose discese glaciali vallive, sappiamo aver costituito una formidabile "collana" di aree di rimaneggiamenti distributivi avvenuti come opportuna risposta ad ogni alternanza di cicli glaciali/interglaciali, fenomeni che hanno favorito, come già ricordato prima, le cladogenesi certamente più recenti di livello specifico o sottospecifico. In altri casi però, soprattutto per spiegare distribuzioni di interi generi o di diverse linee filetiche, si è costretti a pensare a disgiunzioni più antiche, certamente prequaternarie, riconducibili almeno al Miocene medio-superiore, quando non a questo precedenti. Ancora una volta, è forse il caso di ricordare l'importanza che possono avere avuto, localmente, le vicende legate al modellamento morfo-orografico delle valli e della "scarpata" prealpina durante la crisi di salinità del Me- 
diterraneo avvenuta nel Messiniano. L'impostazione generale di molte valli fluviali prealpine, di più lontana origine tettonica, era già in essere in epoca premessiniana, tanto da far conoscere loro alterne fasi di regressione e di trasgressione marina da parte del mare "padano", determinanti complessi processi erosivi e deposizionali. Ma è l'elevata energia del rilievo, esaltata dalla crisi del Mediterraneo, che è responsabile della formazione di profondissime valli - e non solo di quelle attualmente occupate dai ben noti laghi prealpini - solo successivamente colmate da ingenti episodi sedimentari (Bini et al., 1978; Finckh, 1978; Finckh et al., 1984; Cita, 1990). L'incisione dei profondi canyon messiniani ha svolto probabilmente un ruolo non secondario nel determinare i numerosi cladi di livello generico o sopragenerico all'interno di un popolamento che già era insediato nell'area verosimilmente dal Miocene inferiore, se non ancora dall'Oligocene, analogamente a quanto dimostrerebbe l'analisi molecolare eseguita sul popolamento dei Leptodirini dei sistemi montuosi dell'area mediterranea occidentale (Ribera et al., 2010). Risultati databili in tal senso, sia pure ancora con varie incertezze e suscettibili di verifica, ci vengono forniti anche dall'analisi del popolamento alpino di numerosi generi di Carabidi Trechini a costumi sotterranei (Faille et. al., 2013), ma è ipotizzabile, sia pure con le debite differenze dovute alle diverse origini, che anche il popolamento dei principali complessi di Colevidi Leptodirini, gravitanti nelle medesime aree geografiche, sia stato determinato, o quanto meno in qualche misura influenzato, dai medesimi eventi (Giachino, 1993b). Certamente più recenti, come già detto, sarebbero invece i rimaneggiamenti e le sovrapposizioni di areali che hanno determinato l'attuale complessità della situazione distributiva a livello specifico.

\section{RINGRAZIAMENTI}

Per avermi offerto aiuto con la consueta disponibilità sono grato agli amici Massimo Meregalli per la revisione del riassunto in lingua inglese, Achille $\mathrm{Ca}$ sale e Pier Mauro Giachino per la paziente rilettura del manoscritto e per gli utili consigli ricevuti. 


\section{BIBLIOGRAFIA}

Bini A., CitA M.B., GaEtani M., 1978 - Southern alpine lakes: hypothesis of an erosional origin related to the Messinian entrechment. Marine Geology, 27: 271-288.

Caoduro G., Osella G., Ruffo S., 1994 - La fauna cavernicola della Regione Veronese. Memorie del Museo Civico di Storia Naturale, Verona, $2^{\circ}$ s., 11: 144.

Casale A., Giachino P.M., 2010 - Due nuovi Coleotteri ipogei delle Alpi occidentali: Duvalius (Duvalius) lanai n. sp. (Carabidae: Trechini) e Archeoboldoria sturanii n. sp. (Cholevidae: Leptodirinae) (Coleoptera). Rivista piemontese di Storia naturale, 31: 213-240.

Casale A., Vigna Taglianti A., 2005 - Coleotteri Caraboidei delle Alpi e Prealpi centrali e orientali, e loro significato biogeografico. XXXV Congresso della Società Italiana di Biogeografia, Rabbi, 2004. Biogeographia, 26: 129-201.

Chemini C., TAmanini L., 1981 - Considerazioni sulla fauna del Monte Baldo. In: AA. Vv., Il Monte Baldo nei suoi aspetti naturalistici e antropici. Natura alpina, 32, 2a serie (27): 77-82.

Cita M.B., 1990 - I grandi laghi lombardi nel quadro dell'evoluzione neogenica, pp. 60-62. In: SocietÀ GeOlogiCA ITALiAnA (ed) - Guide geologiche regionali. 1. Alpi e Prealpi Lombarde, 292 pp.

Faille A., Casale A., Balke M., Ribera I., 2013 - A molecular phylogeny of Alpine subterranean Trechini (Coleoptera: Carabidae). BMC Evolutionary Biology, 13: 248.

FinCKH P.G., 1978 - Are southern Alpine lakes former Messinian canyons? Geophysical evidence for preglacial erosion in the southern Alpine lakes. Marine Geology, 27: 289-302.

FinCKH P.G., KERRY K., LAMBERT A., 1984 - Seismic stratigraphy and bedrock form in perialpine lakes. Geological Society of America Bulletin, 95: 111-128.

GHIDINI G.M., 1937 - Revisione del genere Boldoria Jeannel (Coleoptera - Bathysciinae). Memorie della Società entomologica italiana, 16: 51-70.

Giachino P.M., 1993a - Canavesiella, nuovo genere di Leptodirinae delle Alpi Occidentali, con due nuove specie (Coleoptera Cholevidae). Bollettino del Museo regionale di Scienze naturali, Torino, 11 (2): 347-363.

Giachino P.M., 1993b - La distribuzione dei generi Binaghites e Bathysciola nelle Alpi Occidentali (Coleoptera Carabidae e Cholevidae). Biogeographia, Lavori della Società Italiana di Biogeografia, (n.s.) 16: 401-424.

Giachino P.M., Vailati D., 1997 - Nuovi dati su Archeoboldoria Ghidini, 1937 con descrizione di A. lanai n. sp. (Coleoptera Cholevidae Leptodirinae). Rivista piemontese di Storia naturale, 18: 161-171.

Giachino P.M., VAILATi D., 2005 - I Cholevidae delle Alpi e Prealpi italiane: inventario, analisi faunistica e origine del popolamento nel settore compreso fra i corsi dei fiumi Ticino e Tagliamento (Coleoptera). XXXV Congresso della Società Italiana di Biogeografia, Rabbi, 2004. Biogeographia, 26: 229-378.

Giachino P.M., VAILATI D., 2008 - Ulteriori considerazioni su alcuni aspetti biologici ed ecologici dell'ambiente sotterraneo. Atti del Seminario Nazionale "Ambiente carsico. I progressi degli studi in Italia sulla soglia del XXI secolo", Bossea, 21-22 maggio 2005: 133-140.

Giachino P.M., Lana E., Vailati D., 2001 - Nuovi dati su Leptodirinae del Piemonte con descrizione di Archeoboldoria pascuttoi n. sp. della Valle Cervo (Coleoptera Cholevidae). Rivista piemontese di Storia naturale, 22: 239-250.

Giachino P.M., Vailati D., CASAle A., 1998 - Major questions in the phylogeny and biogeography of Cholevidae (Coleoptera), with emphasis on the subfamily Leptodirinae, pp. 179-209. In: GiACHINO P.M., PECK S.B. (eds.). Phylogeny and Evolution of Subterranean and Endogean Cholevidae (=Leiodidae Cholevinae). Proceedings of a Symposium (30 August 1996). XX International Congress of Entomology, Florence (Italy), 1996. Atti del Museo Regionale di Scienze naturali, Torino, $296 \mathrm{pp}$.

JeAnNel R., 1924 - Monographie des Bathysciinae. Archives de Zoologie Expérimentale et Générale, 63: 436 pp.

MArAZZi S., 2005 - Atlante orografico delle Alpi. SOIUSA. Suddivisione Orografica Internazionale Unificata del Sistema Alpino. Quaderni di Cultura Alpina. Priuli \& Verlucca (ed.), 82-83: 414 pp.

Minelli A., Ruffo S., 1989 - La fauna cavernicola veneta, pp. 49-60. In: Mietto P., SAuro U. (eds.), Le grotte del Veneto. Regione del Veneto e La Grafica Editrice, Verona: 416 pp.

Perreau M., 2000 - Catalogue des Coléoptères Leiodidae Cholevinae et Platypsyllinae. Mémoires de la Société entomologiques de France, 4: 460 pp.

Perreau M., 2004 - Leiodidae. In: Löbl I., Smetana A. (Eds.). Catalogue of Palaearctic Coleoptera, Vol. 2, Hydrophiloidea Histeroidea - Staphylinoidea. Apollo Books, Stenstrup, pp. 133-203.

PIVA E., 1993 - Lessiniella berica n. sp. dei Monti Berici (Veneto, Vicenza) e osservazioni sul genere (Coleoptera Cholevidae). Bollettino della Società entomologica italiana, 125 (2): 121-130.

PIVA E., 2000 - Contributo alla conoscenza del genere Cansiliella, con descrizione di una nuova specie (Coleoptera Cholevidae). Bollettino della Società entomologica italiana, 132 (2): 123-134. 
Piva E., 2005 - Nuove specie di Orostygia e Oryotus, con note sinonimiche (Coleoptera Cholevidae). Memorie della Società entomologica italiana, 84: 3-44.

PIVA E., 2016 - Miettiella vespertilio nuovo genere nuova specie dei Monti Lessini Veronesi (Veneto, Italia) (Coleoptera Cholevidae Leptodirinae). Bollettino della Società entomologica italiana, 148 (2): 63-70.

Ribera I., Fresneda J., Bucur R., IzQuierdo A., Vogler A. P., Salgado J. M., Cieslak A., 2010 - Ancient origin of a Western Mediterranean radiation of subterranean beetles. BMC Evolutionary Biology, 10: 29.

RuFfo S., 1938 - Studio sulla fauna cavernicola della regione veronese. Istituto di Entomologia della Regia Università di Bologna, 10: 70-116.

RuFFo S., 1950 - Descrizione di due nuovi Catopidi cavernicoli del Veronese e osservazioni sul genere Neobathyscia Müll. Memorie del Museo Civico di Storia Naturale, Verona, 2: 125-133.

Sbordoni V., RAmpini M., Cobolli Sbordoni M., 1982 - Coleotteri Catopidi cavernicoli italiani. Lavori della Società Italiana di Biogeografia, n. s., VII: 253-335.

Sciaky R., Vigna Taglianti A., 1990 - The genus Lessinodytes Vigna Tagliainti, 1982, a biogeographical and systematic puzzle (Coleoptera, Carabidae, Trechinae). Mémoires de Biospéologie, 17: 169-173.

VAILATI D., 1976 - Sulla posizione sistematica di Bathysciola (Hartigiella) baldensis Müller, 1928 con note corologiche, ecologiche e biogeografiche. Natura bresciana, 12: 34-50.

VAILATI D., 1977 - Note corologiche e tassonomiche su alcune specie del genere Boldoria (s.str.) Jeannel. Natura Bresciana, 13: 64-74.

VAILATI D., 1988 - Studi sui Bathysciinae delle Prealpi centro-occidentali. Revisione sistematica, ecologia, biogeografia della "serie filetica di Boldoria" (Coleoptera Catopidae). Monografie di Natura bresciana, 11: 332 pp.

VAILATI D., 1990 - Insubriella paradoxa nuovo genere nuova specie di Bathysciinae delle Prealpi italiane. Natura bresciana, 25: 213-229.

VAILATI D., 1991 - Nuovi dati sulla distribuzione di Pseudoboldoria robiatii (Reitter, 1889) e considerazioni sulla corologia pleistocenica dei Bathysciinae in Lombardia. Natura bresciana, 26: 223-234.

VAILATI D., 1993a - Monguzziella grottoloi nuovo genere nuova specie delle Prealpi Venete (Coleoptera Cholevidae Leptodirinae). Natura bresciana, 28: 261-278.

VAILATI D., 1993b - Segnalazione di una nuova stazione e revisione morfologica di Cryptobathyscia gavardensis Vailati, 1980 (Coleoptera Cholevidae Leptodirinae). Natura bresciana, 28: 279-285.

Vigna TAgLianti A., 1982 - Le attuali conoscenze sui Coleotteri Carabidi cavernicoli italiani. Lavori della società Italiana di Biogeografia, n.s., 7: 339-430.

Vigna Taglianti A., Sciaky R., 1988 - Il genere Lessinodytes Vigna Taglianti, 1982 (Coleoptera, Carabidae, Trechinae). Fragmenta entomologica, 20: 159-180.

ZoIA S., 1998 - Considerations of the present knowledge of the Italian Cholevidae and their distribution, with particular reference to the hypogean species (Coleoptera), pp. 179-209. In: Giachino P.M., Peck S.B. (eds.), Phylogeny and Evolution of Subterranean and Endogean Cholevidae (=Leiodidae Cholevinae). Proceedings of a Symposium (30 August 1996). XX International Congress of Entomology, Florence (Italy), 1996. Atti del Museo Regionale di Scienze Naturali, Torino, 296 pp. 